\title{
Adipocyte Tribbles1 Regulates Plasma Adiponectin and Plasma Lipids in Mice
}

2 Elizabeth E. Ha ${ }^{1}$, Gabriella I. Quartuccia, Ruifeng Ling, Chenyi Xue, Antonio Hernandez-Ono, Rami Imam, Jian Cui, Rajesh K. Soni ${ }^{2}$, Robert C. Bauer ${ }^{1 *}$

${ }^{1}$ Cardiometabolic Genomics Program, Division of Cardiology, Department of Medicine,

Columbia University

${ }^{2}$ Proteomics and Macromolecular Crystallography Shared Resource, Herbert Irving

9 Correspondence should be addressed to:

Robert C. Bauer, PhD

17 Authors have no conflict of interests to report. 


\section{Abstract:}

Multiple GWAS have identified SNPs in the $8 q 24$ locus near the TRIB1 gene that

29 significantly associate with plasma lipids and coronary artery disease. While subsequent studies

30 have uncovered roles for hepatic and myeloid Trib1 in contributing to either plasma lipids or

31 atherosclerosis, the causal tissue for these GWAS associations remains unclear. The same

32 8q24 SNPs significantly associate with plasma adiponectin levels in humans as well, suggesting

33 a role for TRIB1 in adipose tissue. Here, we report that adipocyte-specific Trib1 knockout mice

34 (Trib1_ASKO) have increased plasma adiponectin levels and decreased plasma cholesterol and

35 triglycerides. We demonstrate that loss of Trib1 increases adipocyte production and secretion of

36 adiponectin independent of the known TRIB1 function of regulating proteasomal degradation.

37 RNA-seq analysis of adipocytes and livers from Trib1_ASKO mice suggests that alterations in

38 adipocyte function underlie the plasma lipid changes observed in these mice. Secretomics and

39 RNA-seq analysis revealed that Trib1 ASKO mice have increased production of Lpl and

40 decreased production of Angptl4 in adipose tissue, and fluorescent substrate assays confirm an

41 increase in adipose tissue Lpl activity, which likely underlies the observed triglyceride

42 phenotype. In summary, we demonstrate here a novel role for adipocyte Trib1 in regulating

43 plasma adiponectin, total cholesterol, and triglycerides in mice, confirming previous genetic

44 associations observed in humans and providing a novel avenue through which Trib1 regulates

45 plasma lipids and coronary artery disease. 


\section{Introduction:}

$54 \quad$ Plasma lipids, including triglycerides and cholesterol, are among the strongest risk

55 factors for cardiovascular disease (CVD), and modulation of plasma lipid levels is among the

56 most effective therapeutic strategies at combating atherosclerotic CVD. Multiple genome-wide

57 association studies (GWAS) investigating cardiometabolic risk factors have identified SNPs in

58 the 8q24 genomic locus that associate with plasma triglycerides (TGs), total cholesterol (TC),

59 LDL-cholesterol (LDL-C), HDL-cholesterol (HDL-C), and coronary artery disease (CAD) [1-6],

60 suggesting that this locus contains elements that regulate lipid metabolism and disease risk.

61 These SNPs lie $\sim 40 \mathrm{~kb}$ downstream of the Tribbles1 (TRIB1) gene, which codes for the TRIB1

62 pseudokinase. Studies in multiple genetic mouse models have since confirmed a role for both

63 hepatic and macrophage Trib1 in the regulation of lipid metabolism and CVD [7-9]. Viral

64 mediated liver-specific overexpression of Trib1 in mice was found to decrease plasma

65 cholesterol and TGs, and hepatic deletion of Trib1 increased plasma cholesterol and TG, while

66 also causing hepatic steatosis due to increased de novo lipogenesis [7]. This latter phenotype

67 confirmed an additional GWAS association between the 8q24 SNPs and circulating liver

68 transaminases (ALTs/ASTs) [10], suggestive of a role for TRIB1 in steatosis and hepatocellular

69 health. A more recent study found that myeloid-specific Trib1 knockout mice have reduced

70 atherosclerotic burden [9] due to decreased OxLDL uptake by macrophages and reduced foam

71 cell formation, highlighting the importance of tissue-specific gene functions as well as raising the

72 question of possible roles for Trib1 in other tissues in mediating the GWAS associations.

73 The same SNPs in the 8q24 locus that associate with plasma lipid traits, CAD, and ALTs

74 have been found to additionally associate with plasma adiponectin levels in humans [11]

75 (Supplementary Figure 1). Adiponectin is an adipokine, or signaling molecule secreted from

76 adipocytes, that acts predominantly as an insulin sensitizing agent [12] but can also alter

77 plasma lipids [13], hepatic fat content [14], and even CAD [15]. Given the 8q24 association and

78 the fact that adiponectin is exclusively produced in adipocytes, we hypothesized that TRIB1 
79 plays a role in adipose tissue biology. Additionally, the myriad of roles for adiponectin in

80 regulating cardiometabolic traits begs the question of whether the function of Trib1 in adipocytes

81 is responsible for the observed metabolic genetic associations. Here, we report the first

82 adipocyte-specific Trib1 knockout mouse and show that these mice have increased plasma

83 adiponectin levels and decreased plasma cholesterol and TG levels. Further mechanistic

84 studies reveal that Trib1 regulates plasma adiponectin through increased adiponectin

85 production and secretion, and that Trib1 modulates plasma TG clearance through regulation of

86 adipose-specific lipoprotein lipase (Lpl) activity.

87

88 Results:

89 Adipocyte-specific Trib1 knockout does not alter body weight, adiposity, or adipose inflammation

91 We generated Trib1 adipocyte-specific knockout (Trib1_ASKO) mice by crossing

92 previously described Trib1-floxed (Trib1_fl/fl) C57BL/6 mice [7] with transgenic mice expressing

93 Cre recombinase under the adipocyte-specific Adipoq promoter. Efficient Trib1 deletion in

94 adipose tissue of Trib1_ASKO mice was confirmed by qPCR in both subcutaneous white

95 adipose tissue (scWAT) (Figure 1a) and brown adipose tissue (BAT) (Figure 1b), and we did

96 not detect any compensatory changes in Trib2 or Trib3 expression in scWAT (Figure 1a). Trib1

97 message was unchanged in other tissues, including the livers (Figure 1b) of Trib1_ASKO mice,

98 confirming specificity of the model. Chow-fed Trib1_ASKO mice had similar overall body weight

99 and fat pad mass to Trib1_fl/fl mice (Figure 1c,d), and there was no difference in adipocyte

100 morphology or size as measured by H\&E staining and subsequent morphometric analysis

101 (Figure 1e). Similar results were observed in mice fed a 45\% kcal high-fat diet (HFD) for 12

102 weeks (Figure 1f,g).

103 To better understand the effects of Adipoq-Cre mediated knockout of Trib1 in 104 adipocytes, we utilized an in vitro model of adipocyte culture, where we isolated the stromal 
105 vascular fraction (SVF) from the scWAT of Trib1_fl/fl and Trib1_ASKO mice and differentiated

106 them to adipocytes. Consistent with the lack of an adiposity phenotype in the adult mice, SVF-

107 derived adipocytes from Trib1_fl/fl and ASKO mice differentiated similarly, as assessed by time

108 course gene expression of adipogenic markers Pparg, Cebpa, and Adipoq, as well as by cellular

109 morphology and lipid accumulation (Supplemental Figure 2). Importantly, Trib1 expression in

110 adipocytes derived from ASKO mice was lower relative to adipocytes derived from Trib1_fl/fl

111 mice (Supplemental Figure 2a), demonstrating that Adipoq-Cre is efficiently expressed in the

112 in vitro setting upon differentiation.

113 A previous study reported that Trib1 haploinsufficiency in mice impairs the upregulation

114 of inflammatory genes in adipose in response to proinflammatory stimuli such as LPS, TNF-a,

115 and high-fat diet feeding [16]. Given the known contribution of adipose tissue inflammation to

116 obesity and metabolic disease, we checked if Trib1_ASKO mice had decreased inflammatory

117 markers in adipose. We measured inflammatory gene expression in adipose tissue in both

118 chow-fed and HFD-fed conditions and observed no difference between the groups in either diet

119 setting (Supplemental Figure 3a,b). Similarly, we did not observe any changes in the

120 transcriptional response to TNF-a treatment in SVF-derived adipocytes from Trib1_ASKO mice

121 compared to Trib1_fl/fl controls (Supplemental Figure 3c). These data suggest that the

122 phenotypes we have observed in our mice are not due to changes in adipose inflammation.

124 Adipocyte-specific Trib1 knockout mice have increased plasma adiponectin and

125 decreased plasma lipids

126 Given the association in humans between SNPs near TRIB1 and plasma adiponectin,

127 we first sought to determine if Trib1_ASKO mice had altered circulating adiponectin levels. We

128 found that both male and female Trib1_ASKO mice on chow diet have significantly increased

129 plasma adiponectin (>20\%) levels compared to wild-type counterparts (Figure 2a). The

130 increase in plasma adiponectin was not accompanied by detectable changes in adiponectin 
131 message levels in the scWAT or visceral adipose tissue (VAT) (Figure 2b), suggesting a

132 posttranscriptional role for Trib1 in plasma adiponectin regulation. We checked the plasma

133 levels of other adipokines and found that plasma resistin levels were also increased in

134 Trib1_ASKO mice (Figure 2c). However, plasma levels of leptin, another abundant adipokine,

135 were not significantly changed in Trib1_ASKO mice (Figure 2d), demonstrating that Trib1

136 regulates the secretion of specific adipokines and not global adipokine secretion. Despite

137 increased adiponectin levels, glucose tolerance was not significantly changed in 8-12-week-old

138 chow-fed mice (Figure 2e), and SVF-derived adipocytes did not demonstrate increased insulin

139 signaling upon insulin stimulation (Supplemental Figure 4a). HFD-fed Trib1_ASKO mice

140 maintained increased adiponectin levels (Figure 2f), and, in contrast to chow-fed mice, these

141 mice also had significantly improved glucose tolerance (Figure $\mathbf{2 g}$ ) as well as decreased fasting

142 plasma insulin levels (Supplemental Figure 4b), consistent with studies that show association

143 between increased plasma adiponectin levels and improved insulin sensitivity [12].

144 Since the same SNPs in the 8q24 locus significantly associate with both plasma

145 adiponectin and plasma lipids (LDL-C, HDL-C, and TG), we next asked if adipocyte Trib1

146 contributes to plasma lipid regulation. We found that chow-fed Trib1_ASKO mice display

147 decreased plasma TG (>28\%) and TC (15\%) levels compared to wild-type counterparts (Figure

148 3a,b), demonstrating a role for adipocyte Trib1 in plasma lipid regulation. We note that this

149 phenotype of decreased plasma lipids is the opposite direction of the effect of the liver-specific

150 knockout of Trib1, which results in increased plasma lipids [7], demonstrating opposing tissue-

151 specific roles for Trib1 in regulating plasma lipids. FPLC analysis of pooled plasma revealed

152 that Trib1_ASKO mice have reduced cholesterol in the HDL fraction as well as decreased TGs

153 in both the VLDL and LDL fractions (Figure 3c,d). Trib1_ASKO mice continue to demonstrate

154 lower total plasma cholesterol when placed on HFD for 12 weeks, although TGs normalized to

155 WT levels (Figure 3e,f). To test for involvement of the LDL receptor in the cholesterol

156 phenotype, we crossed the Trib1_ASKO mice to Ldlr KO mice. While plasma TGs did not differ 
157 between the groups (Figure $\mathbf{3 g}$ ), we found that Trib1_ASKO Ldlr KO mice on chow diet had

158 significantly decreased total cholesterol compared to Trib1_fl/fl Ldlr KO mice (Figure 3h),

159 demonstrating that the cholesterol phenotype is at least partially independent of the LDL

160 receptor pathway. FPLC analysis of pooled plasma from Trib1_ASKO; Ldlr KO mice on chow

161 diet further revealed decreased cholesterol levels in both the LDL and HDL fractions in ASKO

162 mice (Figure 3i).

163

164 Trib1 in adipocytes regulates adiponectin secretion in a posttranscriptional and

165 proteasome-independent mechanism

166 Since plasma adiponectin levels were increased in Trib1_ASKO mice, we hypothesized

167 that Trib1 deficiency in adipocytes promotes increased adiponectin secretion. To test this

168 hypothesis, we investigated adiponectin protein expression and secretion from SVF-derived

169 adipocytes from Trib1_fl/fl and Trib1_ASKO scWAT. Consistent with observations in whole

170 adipose tissue, adiponectin mRNA expression was unchanged in SVF-derived adipocytes

171 (Figure 4a). However, adiponectin was increased in the conditioned media above adipocytes

172 derived from Trib1_ASKO SVF compared to Trib1_fl/fl SVF (Figure 4b), confirming increased

173 adiponectin secretion. This was also accompanied by a clear increase in intracellular

174 adiponectin protein levels (Figure 4c), suggesting that increased secretion is in part due to

175 increased cellular adiponectin protein levels, despite the lack of a transcriptional change.

176 We next asked whether Trib1 overexpression in adipocytes would also have an effect on

177 adiponectin secretion and intracellular protein. We generated a doxycycline-inducible 3xFlagHA-

178 tagged Trib1 overexpression 3T3-L1 stable cell line that was able to overexpress Trib1 >100-

179 fold over wild-type values (Figure 4d). We found that Trib1 protein was not detectable in these

180 cells via western blot unless the cells were first treated with the proteasome inhibitor MG132,

181 suggesting that Trib1 is unstable and undergoes rapid proteasomal degradation (Figure 4e) in

182 3T3-L1 cells. To avoid differences in cell line differentiation capacity caused by selection, we 
183 ultimately used lentiviral delivery of Trib1 and eGFP expressed under the CMV promoter in

184 mature 3T3-L1 adipocytes to assess the effects of Trib1 overexpression in culture. We achieved

$185>60$-fold overexpression of Trib1 via this method, but observed no changes in adiponectin

186 secretion or protein levels compared to the GFP control (Figure 4f-i). Thus, while we were able

187 to show that Trib1 deficiency robustly affects adiponectin protein and secretion in adipocytes,

188 we were unable to produce any effect on adiponectin with Trib1 overexpression in vitro.

189 To better understand the molecular function of Trib1 in adipose tissue and how it may be

190 regulating adiponectin, we first investigated previously reported functions of Trib1 described in

191 other models. As a pseudokinase, Trib1 lacks catalytic phosphorylation activity, and is instead

192 understood to function as a scaffolding protein that mediates interactions between its binding

193 partners [17]. In this regard, Trib1 is best known for its role in the proteasomal degradation of

194 the transcription factor C/EBPa via mediating its ubiquitination by the COP1 E3 ubiquitin ligase

195 [18]. In keeping with that function, we found that C/EBPa protein levels were increased in the

196 adipose tissue of Trib1_ASKO mice (Figure 5a) without observable changes in Cebpa message

197 levels (Figure 5b, Supplemental Figure 2c). Although C/EBPa is a known transcriptional

198 regulator of adiponectin expression [19], we did not observe a consistent increase in

199 adiponectin expression in either tissue or SVF-derived adipocytes (Figure 2b, Figure 4a),

200 consistent with Trib1 regulating adiponectin through a mechanism independent of C/EBPa-

201 mediated transcription.

202 Given Trib1's role in mediating ubiquitination of proteins for proteasomal degradation, we

203 further asked if the proteasome was important in Trib1's regulation of C/EBPa and adiponectin.

204 We treated Trib1_ASKO and Trib1_fl/fl SVF-derived adipocytes with MG132 to determine if

205 proteasomal inhibition would increase C/EBPa and adiponectin protein levels in the control cells

206 but not the KO cells, normalizing the protein levels between the two. We found that MG132

207 treatment did normalize C/EBPa protein levels (Figure 5c) between the two groups, consistent

208 with the known function of Trib1 regulating C/EBPa degradation. However, MG132 treatment 
209 did not affect the difference in adiponectin secretion (Figure 5d) or protein levels (Figure 5c)

210 between control and ASKO cells, suggesting that Trib1 is regulating adiponectin through a

211 proteasome and C/EBPa-independent pathway.

213 RNA-sequencing of adipocytes and hepatocytes reveals a primary role for adipose tissue

214 in altered plasma lipid metabolism in Trib1_ASKO mice

215 To understand the mechanism by which adipocyte-specific Trib1 regulates plasma lipids,

216 we sequenced RNA from adipocytes isolated from the scWAT of Trib1_fl/fl and Trib1_ASKO

217 mice. Differential expression analysis revealed over 2000 genes that were differentially

218 expressed at a greater than 2-fold change (Figure 6a), emphasizing a widespread role for Trib1

219 in adipose. We considered the possibility that altered hepatic metabolism could explain the

220 phenotypes observed in Trib1_ASKO mice, given that the liver is a major regulator of lipoprotein

221 metabolism and that adipokines such as adiponectin can signal to the liver. However, RNA-seq

222 of livers from the same mice revealed very few differentially expressed genes, none of which

223 were major lipid regulators (Figure 6b, Supplemental Table S1). Thus, we concluded that

224 adipocyte Trib1 is regulating plasma lipids through direct regulation by adipose tissue itself.

225 We next ranked differentially expressed genes by signal-to-noise ratio in expression and

226 performed gene set enrichment analysis (GSEA) (Figure 6c). Notably, GSEA highlighted a

227 striking enrichment of mitochondrial genes among upregulated genes, including genes coding

228 for proteins in the electron transport chain, mitochondrial ribosomes, and the mitochondrial

229 membrane, suggesting a potential role for mitochondria in the phenotypes we observed.

230 Furthermore, multiple gene sets involved in lipid metabolism were upregulated, pointing towards

231 a role for adipocyte-specific Trib1 in regulation of lipids through lipid breakdown and metabolism

232 (Supplemental Table S2).

233

234 Lipoprotein lipase activity is increased in Trib1_ASKO adipose 
Given the importance of adipocytes in TG storage, we next sought to identify the

236 physiological mechanism whereby adipocyte-specific Trib1 regulates plasma TGs. One

237 mechanism through which adipose contributes to plasma TGs is through the lipolysis of TGs in

238 the lipid droplet and their release as free fatty acids into the bloodstream; subsequently, free

239 fatty acids can be repackaged as TGs and secreted by the liver in the form of VLDL [20]. To

240 assess for an effect on lipolysis by deletion of Trib1 in adipose, we first measured plasma

241 nonesterified free fatty acids (NEFA) and glycerol, markers of lipolysis, after stimulating lipolysis

242 by fasting mice overnight for $16 \mathrm{hr}$. We found that NEFA and glycerol levels were comparable

243 between Trib1_ASKO and Trib1_fl/fl mice after prolonged fasting (Supplemental Figure 5a,b),

244 suggesting that loss of adipocyte Trib1 does not impact lipolysis rates under stimulation. We

245 also assessed the activation of hormone sensitive lipase (HSL), a key lipolytic driver in adipose

246 that is activated by phosphorylation of key residues, and were unable to detect a difference

247 between phospho-HSL in subcutaneous adipose from Trib1_ASKO and Trib1_fl/fl mice

248 (Supplemental Figure 5c,d). Consistent with these observations, VLDL secretion also was

249 unchanged (Supplemental Figure 5e), suggesting that the adipose is not providing significantly

250 different loads of fatty acids to the liver.

251 Given the observed changes in plasma adipokine secretion and the importance of

252 adipose endocrine functions, we next performed an unbiased secretomics experiment to identify

253 differentially secreted proteins from Trib1_ASKO adipose. We incubated scWAT explant tissue

254 from Trib1_fl/fl and Trib1_ASKO mice for 6 hours in serum-free media, and then identified and

255 quantified proteins in the conditioned media via data independent acquisition (DIA)

256 (Supplemental Table S3). Consistent with our earlier findings of increased adiponectin and

257 resistin (Figure 2a,c), an increase in adiponectin (>50\%) and resistin was found in the

258 conditioned media from the ASKO tissue (Figure 7a), thus validating our secretomics data.

259 Interestingly, we observed significantly decreased Angptl4 secretion and a trend towards

260 increased Lipoprotein lipase (Lpl) secretion from Trib1_ASKO scWAT explants (Figure 7a). 
261 Angptl4 is an inhibitor of Lpl, which binds to the endothelium in vasculature and hydrolyzes TGs

262 in circulating lipoproteins to free fatty acids, allowing for their uptake and clearance into tissues,

263 including adipose [21, 22]. In addition to changes in secretion, $L p /$ expression was increased

264 and Angpt/4 expression was decreased significantly in our RNA-seq dataset. The expression of

265 Lmf1, which codes for Lipase maturation factor and is important for the proper folding and

266 secretion of Lpl [23], was also notably increased in ASKO adipocytes (Figure 7b). Overall,

267 these suggest increased Lpl activity in ASKO adipose tissue. We next measured Lpl activity in

268 adipose tissue extracts from Trib1_fl/fl and Trib1_ASKO mice via cleavage of a fluorescent lipid

269 substrate and found that adipose tissue extracts from both scWAT and VAT from Trib1_ASKO

270 mice demonstrated increased lipase activity (Figure 7c,d), likely contributing to increased TG

271 clearance in Trib1_ASKO mice.

Discussion:

Genome-wide association studies have identified SNPs near the TRIB1 gene that

275 significantly associate with plasma lipids and CAD, and previous work in liver-specific and

276 macrophage-specific mouse models have shown important roles for Trib1 in plasma lipid

277 regulation as well as in hepatic lipogenesis [7]. An additional GWAS showing an association

278 between the SNPs and circulating adiponectin levels [11] suggested a potential role for

279 adipocyte-specific TRIB1 in lipid metabolism, and we report here that adipocyte-specific Trib1

280 knockout mice have increased plasma adiponectin as well as decreased plasma cholesterol and

281 TGs, thus validating novel roles for adipocyte-specific Trib1 in both plasma adiponectin and lipid

282 regulation. Interestingly, the reduction in plasma TGs and cholesterol in Trib1_ASKO mice is the

283 opposite of the previously reported liver-specific knock out mice [7], which exhibited increased

284 plasma TC and TG. This suggests tissue-specific roles for TRIB1 in regulating plasma lipid

285 metabolism, and also highlights the difficulty in determining the causal tissue for associations

286 found in GWAS. Given that there is currently no known functional link between the 8q24 GWAS 
287 SNPs and TRIB1 expression or function [24], further functional genomic studies will be required

288 to understand if and how these SNPs contribute to tissue-specific TRIB1 function.

TRIB1 is one of three mammalian homologs of the Tribbles pseudokinase that was first

290 discovered in Drosophila [17]. These proteins bear homology to serine/threonine kinases, but

291 lack key catalytic residues that render them unable to catalyze phosphorylation. Instead, they

292 are best understood to function as scaffolding proteins that bring other proteins into proximity

293 with each other to mediate signaling events [17]. One of the best understood molecular

294 functions for TRIB1 is its role in mediating the ubiquitination and degradation of the transcription

295 factor C/EBPa by bringing it into proximity of the COP1 E3 ubiquitin ligase. Tribbles-mediated

296 regulation of C/EBPa protein levels has been shown to be an important function in several

297 models, including as a causal mechanism for the hepatic lipogenesis phenotype in LSKO mice

298 [7], for myeloid cell proliferation in the context of leukemia [18], and in oogenesis in drosophila

299 [25]. We report here that Trib1_ASKO adipocytes also exhibit increased C/EBPa protein levels

300 in the absence of any change in gene expression, and that C/EBPa protein levels are

301 normalized between control and Trib1_ASKO SVF-derived adipocytes under conditions of

302 proteasomal inhibition. Thus, we provide evidence that adipocyte Trib1 also regulates C/EBPa

303 through proteasomal degradation.

$304 \mathrm{C} / \mathrm{EBPa}$ is a critical regulator of adipocyte differentiation [26]. However, despite

305 increased C/EBPa protein, we interestingly did not observe any differences in adiposity or

306 adipose morphology in Trib1_ASKO mice, or in the in vitro differentiation of adipose stem cells

307 from the ASKO mice. This could be a result of the Adipoq promoter-driven Cre, which is induced

308 late in the process of adipocyte differentiation, thus making our mouse model a post-

309 differentiation knockout of adipocyte Trib1. A previous report showed that Trib1 overexpression

310 can inhibit differentiation of 3T3-L1 cells [27], providing precedent for a role for Trib1 in

311 adipogenesis. Further studies utilizing a different Cre transgene would be required to determine

312 if Trib1 has a similar role in regulating adipogenesis in vivo. 
We also found that Trib1_ASKO adipocytes have both increased cellular levels of

314 adiponectin and increased secretion of adiponectin, with the former likely driving the latter. We

315 observed no change in Adipoq gene expression via repeated qPCR measurements in multiple

316 ex vivo cell culture experiments, whole adipose tissue, and isolated adipocytes from

317 Trib1_ASKO mice. Thus, the increase in adiponectin protein comes in the absence of any

318 reliable change in Adipoq gene expression, suggesting a post-transcriptional mechanism of

319 regulation. We will note, however, that Adipoq expression was surprisingly increased in

320 Trib1_ASKO mice in our RNA-seq dataset (padj $=0.011$, fold change $=1.33$ ). This raises the

321 possibility that increased protein levels of $\mathrm{C} / \mathrm{EBPa}$, which is a well-known transcriptional

322 regulator of adiponectin expression [19], or a different unknown transcription factor is driving a

323 small increase in Adipoq gene expression that qPCR is not sensitive enough to reliably

324 measure. We note though that while MG132 treatment of SVF-derived adipocytes increases

$325 \mathrm{C} / \mathrm{EBPa}$ protein levels, it actually decreases the secretion of adiponectin in wild-type SVF-

326 derived adipocytes (Figure 5d). Thus, while Trib1 certainly appears to regulate C/EBPa in

327 adipose, we propose this is a separate mechanism from the one governing Trib1 regulation of

328 adiponectin. The exact nature of the relationship between Trib1 and cellular adiponectin levels

329 remains to be determined.

330 As noted, Trib1_ASKO mice exhibit decreased plasma TC and TG levels. We

331 subsequently determined that these mice also have increased adipose Lpl activity, likely driving

332 increased uptake of plasma TGs into adipocytes and contributing to the reduction in plasma TG.

333 This might be expected to drive an increase in adipocyte size, which we did not observe in

334 Trib1_ASKO mice. However, GSEA of our RNA-seq data revealed upregulation of genes

335 encoding mitochondrial components, suggestive of increased mitochondrial activity. A resulting

336 increase in energy expenditure could potentially explain the lack of an adipocyte size phenotype

337 in Trib1_ASKO mice despite increased adipose tissue Lpl activity and presumed fatty acid

338 uptake. Notably, C/EBPa regulates genes involved in lipid metabolism in adipose tissue and is a 
339 known transcriptional regulator of $L p l[28,29]$, and polymorphisms in C/EBPa have also been

340 found to associate with plasma TG levels in humans [29]. It is thus possible that increased

341 C/EBPa protein levels in Trib1_ASKO adipose may contribute to this lipid phenotype. There is

342 some precedent that the increased Lpl activity in the Trib1_ASKO could contribute to the

343 observed decrease in plasma cholesterol. Multiple studies using transgenic Lpl animal models

344 [30-32] as well as Angptl4 knockout or transgenic mice [33, 34] demonstrate that increased Lpl

345 activity protects from diet-induced hypercholesterolemia and decreases plasma LDL-C levels,

346 though the effects are not as robust as effects on plasma TGs. Mechanistically, lipolysis-

347 mediated reductions in TGs in VLDL particles have been proposed to facilitate enhanced

348 clearance of the resulting remnant particles via receptors such as the LDLR [35, 36]. Further

349 studies are necessary to determine if increased LPL activity is responsible for the cholesterol

350 phenotype in Trib1_ASKO mice.

351 Adiponectin has many well-studied roles in regulating cardiometabolic traits, including

352 lipid metabolism and coronary artery disease $[13,15]$. Thus, an important outstanding question

353 is whether adiponectin is driving the lipid phenotypes observed in the Trib1_ASKO mice.

354 Adiponectin's role in insulin sensitization is perhaps its most widely recognized physiological

355 effect $[37,38]$, and indeed we found that Trib1_ASKO mice demonstrated improved glucose

356 tolerance compared to Trib1_fl/fl mice when placed on high-fat diet. However, a role for

357 adiponectin in regulating plasma cholesterol is less clear. One previous report using adiponectin

358 transgenic mice with 10-fold increased adiponectin levels found decreased cholesterol in those

359 mice [39]. In humans, numerous epidemiological studies have been conducted looking at

360 associations between adiponectin and plasma LDL-cholesterol, yet many of these are

361 conflicting or inconclusive [13]. Epidemiological studies and genetic mouse models do provide

362 clear support for a role for adiponectin in TG and VLDL metabolism. In particular, plasma

363 adiponectin correlates with decreased plasma TGs and increased HDL-C in humans [40], and

364 adiponectin transgenic mice with 3-fold increased plasma adiponectin levels have increased Lpl 
365 expression and activity in adipose tissue as well as increased TG clearance [41]. Thus, although

366 the adiponectin phenotype in Trib1_ASKO mice is mild ( 20-30\% increase) compared to

367 transgenic mouse models, it is possible that the increased adipose tissue Lpl activity we

368 observe in Trib1_ASKO mice is secondary to increased adiponectin levels. Further studies will

369 be necessary to determine if the adiponectin phenotype is required for the observed changes in

370 Trib1_ASKO plasma lipids.

371 Overall, our studies show that adipocyte-specific Trib1 is a negative regulator of

372 adiponectin secretion, and that this appears to be through a C/EBPa-independent mechanism.

373 Furthermore, we show that adipocyte-specific Trib1 regulates plasma lipids in a direction

374 opposite to that of the previously studied LSKO model, and that regulation of TG clearance via

375 adipose Lpl in part explains the decreased plasma TG levels in ASKO mice. In contrast to

376 hepatic Trib1, these data suggest a therapeutically beneficial effect of reduced adipocyte Trib1

377 activity, underscoring the continued importance of further studies on Trib1 and the 8q24 lipid

378 and CAD GWAS locus.

381 Methods:

382 Animals

383 The previously reported Trib1_fl/fl mice (Bauer et al, JCl 2015) were bred in house. 384 Adipoq-Cre mice (stock\#010803) and Ldlr KO mice (stock\#002207) were obtained from 385 Jackson Labs. Mice were fed ad-libitum on chow diet unless otherwise noted. All experiments 386 were performed when mice were 8-12 weeks old. Mice were fasted for $4 \mathrm{hr}$ prior to collecting 387 plasma samples, unless stated otherwise. Blood was collected retro-orbitally and spun at 388 10,000 rpm for $7 \mathrm{~min}$. Fasting cholesterol and TGs were measured via plate assay using Infinity 389 reagents (Fisher TR13421 and TR22421), and adipokine levels were measured via ELISA 390 (adiponectin: Millipore EZ-MADPK, leptin: Millipore EZML-82K, resistin: R\&D MRSN00). For 
391 HFD experiments, mice were placed on 45\% kcal HFD (Research Diets D12451) starting at 8-

39212 weeks of age. Plasma was collected retro-orbitally at $0,4,8$, and 12 weeks of HFD, and

393 glucose tolerance testing performed at 0, 6, and 12 weeks of HFD as previously described [42].

394 For fasting/refeeding experiments, mice were fasted overnight for $16 \mathrm{hr}$ and then fed ad-libitum

395 with chow diet for 3hr. To measure in vivo TG secretion, plasma triglycerides were measured in

3964 hr-fasted mice 30, 60, 120, and 180 min after i.p. injection of $1 \mathrm{mg}$ pluronic (P407) per gram

397 mouse body weight. All in vivo studies described here were approved by Columbia University’s

398 Institutional Animal Care and Use Committee prior to commencement.

$400 \quad$ FPLC analysis of pooled plasma

$401200 \mu \mathrm{l}$ of pooled plasma from gender and genotype matched mice $(n=4-9)$ was loaded

402 onto a Superose 6 column (GE Healthcare) calibrated with elution buffer $(0.15 \mathrm{M} \mathrm{NaCl}, 1 \mathrm{mM}$

403 EDTA). The lipoproteins were eluted in a total of $20 \mathrm{~mL}$ elution buffer in $0.5 \mathrm{~mL}$ fractions at a

404 rate of $0.3 \mathrm{~mL} / \mathrm{min}$. The cholesterol and TG content of each fraction was determined by plate 405 assay.

407 Western Blot Analysis

408 Tissues or cells were lysed and homogenized in RIPA buffer supplemented with $1 \mathrm{x}$ Halt

409 Protease and Phosphatase inhibitor (Fisher Scientific P178444). The lysate was centrifuged at

$41012,000 \mathrm{xg}$ for $15 \mathrm{~min}$ at $4 \mathrm{oC}$ to clarify the protein prep from cellular debris and lipids. $\sim 30 \mathrm{ug}$

411 protein was loaded onto $10 \%$ bis-tris SDS-PAGE gel and transferred onto a nitrocellulose

412 membrane. The membrane was blocked in either $5 \%$ milk or BSA (for phospho-protein analysis)

413 and incubated in the appropriate primary antibody (adiponectin (R\&D AF1119), Trib1 (Millipore

414 09-126), Flag (Sigma F7425), Tubulin (CST 3873S), C/EBPa (CST 2295S), Beta-actin (Santa

415 Cruz sc-81178), Hsl, pHsl565, pHsl563, and pHsl660 (CST 8334T)) overnight. Protein was

416 detected using a secondary HRP-linked antibody and Luminata Classico Western HRP 
417 Substrate (Millipore WBLUC0020). To reprobe membranes, membranes were incubated in

418 stripping buffer (Fisher Scientific PI21059) for 15 min before reblocking.

$420 \quad$ qPCR analysis

421 RNA from tissues and cells were isolated using the RNeasy Mini kit (Qiagen). cDNA was

422 synthesized using the High-Capacity cDNA Reverse Transcription Kit (Applied Biosystems).

423 qPCR was performed using predesigned Taqman probes from Thermo Fisher Scientific. Gene

424 expression data was normalized to Gapdh and presented as fold change relative to the

425 Trib1_fl/fl control group (exceptions indicated in the figure legend).

427 Microscopy

428 For adipose tissue histology, scWAT samples $(<4 \mathrm{~mm}$ thick) from Trib1_fl/fl and

429 Trib1_ASKO mice were fixed in 4\% PFA for $24 \mathrm{hr}$. The tissues were then embedded in paraffin, 430 sectioned at $7 \mu \mathrm{m}$, and H\&E stained. For each mouse, 4 sections at $70 \mu \mathrm{m}$ intervals were 431 imaged on a Nikon Eclipse Ti microscope with the 40x objective and analyzed using the

432 Adiposoft ImageJ plugin (parameters: minimum diameter $=10 \mu \mathrm{m}$, maximum diameter $=100$ $433 \mu \mathrm{m})$. For Oil Red O staining, cells were fixed in 4\% PFA for $15 \mathrm{~min}$ and then placed in $0.3 \% \mathrm{w} / \mathrm{v}$ 434 Oil Red O in $60 \%$ isopropanol for $30 \mathrm{~min}$. The cells were washed $5 \mathrm{X}$ in distilled $\mathrm{H} 2 \mathrm{O}$, and then 435 imaged with the $20 x$ objective.

437 RNA-seq of adipocytes and hepatocytes

438 8-12-week-old male mice were euthanized and perfused with PBS after a $4 \mathrm{hr}$ fast.

439 Subcutaneous inguinal fat pads from individual mice were harvested, minced, and then placed 440 in $6 \mathrm{~mL}$ digestion media $(0.14 \mathrm{U} / \mathrm{mL}$ Liberase TM, $50 \mathrm{U} / \mathrm{mL}$ DNAse I, $20 \mathrm{mg} / \mathrm{mL}$ BSA in DMEM) 441 for $1 \mathrm{hr}$ at $37 \mathrm{oC}$, shaking at $250 \mathrm{rpm}$. The tissue prep was then filtered through a $100 \mu \mathrm{m}$ cell 442 strainer, and spun at $300 \mathrm{xg}$ for $10 \mathrm{~min}$. The floating white layer was collected as the adipocyte 
443 fraction and placed in $1 \mathrm{~mL}$ Qiazol. RNA was then isolated using the RNeasy Lipid Tissue Mini

444 Kit (Qiagen). Livers from the same mice were harvested and homogenized in Trizol, and RNA

445 was isolated via chloroform extraction. RNA quality was assessed via BioAnalyzer before being

446 submitted to the core for bulk, paired-end RNA-sequencing (NextSeq 500). Reads were aligned

447 using STAR and featurecounts, and differential expression analysis was performed using the

448 DESeq2 package. Differentially expressed genes (padj < 0.050) were ranked by Signal-to-noise

449 ratio of median normalized counts and analyzed by GSEA using the Gene Ontology gene sets

450 (c5.go.v7.2.symbols.gmt) from MSigDB, using gene set size $\leq 200$ and 1000 permutations of

451 the gene sets to determine enrichment score. Cytoscape enrichment plots were constructed

452 from GSEA results using FDR < 0.01, and a combined coefficient $>0.375$ with combined

453 constant 0.5 as described in [43]. Nodes were clustered using the MCL clustering algorithm in

454 the Autoannotate Cytoscape App. Annotations of clusters were manually curated.

455

SVF Generation and Differentiation

457 Subcutaneous inguinal fat pads from 3-5 mice of the same gender and genotype were 458 combined and minced in digestion buffer (L-15 Leibovitz media, 1.5\% BSA, 1\% Pen/Strep, 10 $459 \mathrm{U} / \mathrm{mL}$ DNasel, $480 \mathrm{U} / \mathrm{mL}$ Hyaluronidase, $0.14 \mathrm{U} / \mathrm{mL}$ Liberase TM). Tissue was allowed to 460 dissociate in digestion buffer for $1 \mathrm{hr}$ at $37 \mathrm{oC}$, shaking at $250 \mathrm{rpm}$. The tissue prep was then 461 filtered through a $100 \mu \mathrm{M}$ cell strainer and spun at $300 \mathrm{xg}, 40 \mathrm{C}$, for $10 \mathrm{~min}$. The pellet was 462 saved and resuspended in $10 \mathrm{~mL}$ culture medium (DMEM, 10\% FBS, 1\% Pen/Strep, 2 mM L463 Glut). The cells were spun at $300 \mathrm{xg}, 4 \mathrm{oC}$, for $10 \mathrm{~min}$, and resuspended in $5 \mathrm{~mL}$ culture 464 medium supplemented with $1 \mu \mathrm{g} / \mathrm{mL}$ insulin before seeding. Media was changed every $2-3$ 465 days until the cells were $>95 \%$ confluent. Differentiation was initiated with a cocktail including 466 10\% FBS, 1\% Pen/Strep, $5 \mu \mathrm{g} / \mathrm{mL}$ insulin, $1 \mu \mathrm{M}$ Rosiglitazone, $1 \mu \mathrm{M}$ Dexamethasone, and 250 $467 \mu \mathrm{M}$ IBMX in DMEM/F12. After $48 \mathrm{hr}$, cells were maintained in DMEM/F12 supplemented with 
468 only $10 \%$ FBS, $1 \%$ Pen/Strep, $5 \mu \mathrm{g} / \mathrm{mL}$ insulin, and $1 \mu \mathrm{M}$ Rosiglitazone. Experiments were 469 started after day 7 of differentiation.

471 Global quantitative proteomics of Explant secretomics

472 Mice were euthanized and perfused with PBS before dissection of subcutaneous

473 adipose fat pads. $50 \mathrm{mg}$ of tissue was placed into $1 \mathrm{~mL}$ of warm, serum-free DMEM in a 12 well

474 plate and pinned down with transwell insert. The media was collected after 6hr and protein was

475 precipitated using methanol. DIA (Data independent acquisition) based proteomics was used. In

476 brief, protein precipitated pellets were resuspended in SDC lysis buffer [44] ( $1 \%$ SDC, $10 \mathrm{mM}$

477 TCEP, $40 \mathrm{mM} \mathrm{CAA}$ and $100 \mathrm{mM}$ Tris- $\mathrm{HCl} \mathrm{pH} \mathrm{8.5)} \mathrm{and} \mathrm{boiled} \mathrm{for} 10 \mathrm{~min}$ at $95^{\circ} \mathrm{C}, 1500 \mathrm{rpm}$ to

478 denature and reduce and alkylate cysteins, followed by sonication in a water bath, cooled down

479 to room temperature. Protein concentration was estimated by BCA measurement and $20 \mu \mathrm{g}$

480 were further processed for overnight digestion by adding LysC and trypsin in a 1:50 ratio ( $\mu$ g of

481 enzyme to $\mu \mathrm{g}$ of protein) at $37^{\circ} \mathrm{C}$ and $1500 \mathrm{rpm}$. Peptides were acidified by adding $1 \%$ TFA,

482 vortexed, and subjected to StageTip clean-up via SDB-RPS. $20 \mu \mathrm{g}$ of peptides were loaded on

483 two 14-gauge StageTip plugs. Peptides were washed two times with $200 \mu \mathrm{L} 1 \%$ TFA $99 \%$ ethyl

484 acetate followed $200 \mu \mathrm{L} 0.2 \% \mathrm{TFA} / 5 \% \mathrm{ACN}$ in centrifuge at $3000 \mathrm{rpm}$, followed by elution with

$48560 \mu \mathrm{L}$ of $1 \%$ Ammonia, $50 \%$ ACN into eppendorf tubes and dried at $60^{\circ} \mathrm{C}$ in a SpeedVac 486 centrifuge. Peptides were resuspended in $10 \mu \mathrm{L}$ of $3 \%$ acetonitrile $/ 0.1 \%$ formic acid and injected 487 on Thermo Scientific ${ }^{\mathrm{TM}}$ Orbitrap Fusion ${ }^{\mathrm{TM}}$ Tribrid $^{\mathrm{TM}}$ mass spectrometer with DIA method [45] for 488 peptide MS/MS analysis. The UltiMate 3000 UHPLC system (Thermo Scientific) and EASY489 Spray PepMap RSLC C18 50 cm x $75 \mu \mathrm{m}$ ID column (Thermo Fisher Scientific) coupled with 490 Orbitrap Fusion (Thermo) were used to separate fractioned peptides with a 5-30\% acetonitrile 491 gradient in $0.1 \%$ formic acid over $127 \mathrm{~min}$ at a flow rate of $250 \mathrm{~nL} / \mathrm{min}$. After each gradient, the 492 column was washed with $90 \%$ buffer B for 5 min and re-equilibrated with $98 \%$ buffer A $(0.1 \%$ 493 formic acid, 100\% HPLC-grade water) for 40min. Survey scans of peptide precursors were 
494 performed from $350-1200 \mathrm{~m} / \mathrm{z}$ at $120 \mathrm{~K}$ FWHM resolution (at $200 \mathrm{~m} / \mathrm{z}$ ) with a $1 \times 10^{6}$ ion count

495 target and a maximum injection time of $60 \mathrm{~ms}$. The instrument was set to run in top speed mode

496 with 3 s cycles for the survey and the MS/MS scans. After a survey scan, $26 \mathrm{~m} / \mathrm{z}$ DIA segments

497 will be acquired at from $200-2000 \mathrm{~m} / \mathrm{z}$ at $60 \mathrm{~K}$ FWHM resolution (at $200 \mathrm{~m} / \mathrm{z}$ ) with a $1 \times 10^{6}$ ion

498 count target and a maximum injection time of $118 \mathrm{~ms}$. HCD fragmentation was applied with $27 \%$

499 collision energy and resulting fragments were detected using the rapid scan rate in the Orbitrap.

500 The spectra were recorded in profile mode. DIA data were analyzed with directDIA 2.0 (Deep

501 learning augmented spectrum-centric DIA analysis) in Spectronaut Pulsar X, a mass

502 spectrometer vendor independent software from Biognosys. The default settings were used for

503 targeted analysis of DIA data in Spectronaut except the decoy generation was set to "mutated".

504 The false discovery rate (FDR) will be estimated with the mProphet approach and set to $1 \%$ at

505 peptide precursor level and at $1 \%$ at protein level.

506 Results obtained from Spectronaut were further analyzed using the Spectronaut

507 statistical package. Significantly changed protein abundance was determined by un-paired t-test

508 with a threshold for significance of $p<0.05$ (permutation-based FDR correction) and 0.58

$509 \log 2 \mathrm{FC}$.

512 Cloning and Lentivirus Production

513 Lentiviral constructs for tetracycline-inducible expression of proteins (mTrib1 and eGFP)

514 in adipocytes for overexpression experiments were cloned by first introducing a $3 \times$ FlagHA tag at

515 the C-terminal end of each protein. The fusion proteins were then cloned into the pEN-TTMCS

516 entry vector to introduce a tight TRE promoter and subsequently cloned into the pSLIK-neo

517 lentiviral plasmid via Gateway cloning. mTrib1 and eGFP were also cloned into the

518 pLentiCMVPuroDEST lentiviral vector for constitutive overexpression under the CMV promoter. 
To produce the virus, $5 \times 10^{6} 293$ T cells were seeded in T75 flasks and transfected with

$5202 \mu \mathrm{g} \mathrm{MD} 2 \mathrm{G}, 3 \mu \mathrm{g} \mathrm{Pax2}$, and $5 \mu \mathrm{g}$ lentiviral construct with $30 \mu \mathrm{L}$ Fugene 6 (Promega) the

521 following day. The media was changed the day after transfection, and the viral supernatant was

522 collected and pooled after $24 \mathrm{hr}$ and $48 \mathrm{hr}$. The supernatant was filtered through a $0.45 \mu \mathrm{m}$ filter,

523 aliquoted, and stored at $-800 \mathrm{C}$ until use.

524

525 Adipocyte Cell culture

526 3T3-L1 cells were purchased from ATCC and cultured in DMEM supplemented with 10\%

527 FBS, $1 \mathrm{mM}$ Sodium Pyruvate, and 1\% Pen/Strep. Cells were tested for mycoplasma every three

528 months. To differentiate 3T3-L1 cells to adipocytes, cells were induced with growth media

529 supplemented with $1 \mu \mathrm{M}$ Dexamethasone, $0.5 \mathrm{mM}$ IBMX, and $1 \mu \mathrm{g} / \mathrm{mL}$ Insulin for $48 \mathrm{hr}$, and

530 then maintained in growth media supplemented with only $1 \mu \mathrm{g} / \mathrm{mL}$ insulin. Experiments were

531 typically performed on cells 7 - 9 days after differentiation induction. Stable doxycycline-

532 inducible 3T3-L1 cells were generated by transducing cells with lentivirus at an MOI 100 ,

533 followed by selection with $1.5 \mu \mathrm{g} / \mathrm{mL}$ puromycin. Conditioned media was collected in OptiMEM I

534 reduced serum media.

535

Fluorescent LPL Assay

537 The Lpl activity assay was adapted from Basu et. al [46]. Briefly, adipose tissue was

538 minced in $5 \mu \mathrm{l} \times \mathrm{mg}$ tissue weight volume in tissue incubation buffer (PBS, $2 \mathrm{mg} / \mathrm{ml} \mathrm{FA}$-free

539 BSA, $5 \mathrm{U} / \mathrm{mL}$ heparin), incubated for $1 \mathrm{hr}$ in a $37 \mathrm{oC}$ shaker, and centrifuged at 3,000 rpm for 15

$540 \min$ at 4 oC. The clarified supernatant was placed in fresh tubes and diluted 1:10 in tissue

541 incubation buffer. $4 \mu$ of lysate was placed in duplicate in a black-walled 96-well plate, and 100

$542 \mu \mathrm{L}$ reaction buffer $(0.15 \mathrm{M} \mathrm{NaCl}, 20 \mathrm{mM}$ Tris- $\mathrm{HCl}$ pH 8.0, 0.0125\% Zwittergent, 1.5\% FA-free

543 BSA, $0.62 \mu \mathrm{M}$ EnzChek (Invitrogen E33955)) was added to each well. The reaction was allowed

544 to incubate $20 \mathrm{~min}$ at $37 \mathrm{oC}$, and was then read at an excitation of 485 and emission of 515 . A 
545 blank RFU value was subtracted from all experimental RFU values, and the resulting values

546 were reported.

548 Statistics

GraphPad Prism 8 was used to graph data and to perform parametric 2-tailed Student's t

550 tests and 1- and 2-way ANOVA analyses with multiple correction using either Dunnett's,

551 Sidak's, or Tukey's method as indicated in the figure legends.

552

553

554 Acknowledgements: These studies were funded by R01HL141745 (R.C.B) from the

$555 \mathrm{NIH} / \mathrm{NHLBI}$ and Scientific Development Grant 16SDG31180039 (R.C.B) from the American

556 Heart Association. Additionally, E.E.H was supported by a Ruth L. Kirschstein Individual

557 Predoctoral F30 NRSA (F30HL146076-01A1) from the NIH/NHLBI.

558

559 Author Contributions: R.C.B conceived the project, designed the experiments, supervised

560 analyses and edited the manuscript. E.E.H. performed the majority of the experiments and data

561 analysis, and wrote the first draft of the manuscript and edited subsequent versions. G.I.Q

562 helped establish the mouse colony, assisted with SVF isolation, and performed related

563 molecular biology (i.e. cloning, western blots). R.L. performed western blotting for adipose

564 lipolysis proteins and ELISA analysis. C.X. performed the DESeq2 analysis of the RNA-seq data.

565 A.H. performed initial FPLC analysis and assisted with all FPLC analysis. R.I. performed SVF

566 isolation and cloning of viral vectors. J.C. managed the animal colony and assisted with insulin

567 trait experiments. R.K.S. performed and analyzed the secretomics MS experiment. All the

568 authors read and approved the manuscript.

569

570 Competing Interests Statement: The Authors declare no competing interests. 
572 Data Availability Statement: GEO accession numbers for RNA-seq data will be available prior

573 to publication. Full list of identified proteins and differentially secreted proteins from DIA

574 secretomics (Figure 7a) is available in supplemental table S3. Other data that support the

575 findings of this study are available from the corresponding author upon reasonable request.

576

577 References:

578

579 1. Willer, C.J., et al., Newly identified loci that influence lipid concentrations and risk of $580 \quad$ coronary artery disease. Nat Genet, 2008. 40(2): p. 161-9.

$581 \quad$ 2. Kathiresan, S., et al., Six new loci associated with blood low-density lipoprotein

582 cholesterol, high-density lipoprotein cholesterol or triglycerides in humans. Nat Genet,

583 2008. 40(2): p. 189-97.

584 3. Teslovich, T.M., et al., Biological, clinical and population relevance of 95 loci for blood $585 \quad$ lipids. Nature, 2010. 466(7307): p. 707-13.

586 4. Consortium, I.K.C., Large-scale gene-centric analysis identifies novel variants for 587 coronary artery disease. PLoS Genet, 2011. 7(9): p. e1002260.

$588 \quad 5 . \quad$ Consortium, C.A.D., et al., Large-scale association analysis identifies new risk loci for 589 coronary artery disease. Nat Genet, 2013. 45(1): p. 25-33.

$590 \quad 6 . \quad$ Willer, C.J., et al., Discovery and refinement of loci associated with lipid levels. Nat 591 Genet, 2013. 45(11): p. 1274-1283.

592 7. Bauer, R.C., et al., Tribbles-1 regulates hepatic lipogenesis through posttranscriptional 593 regulation of C/EBPalpha. J Clin Invest, 2015. 125(10): p. 3809-18.

594 8. Burkhardt, R., et al., Trib1 is a lipid-and myocardial infarction-associated gene that 595 regulates hepatic lipogenesis and VLDL production in mice. J Clin Invest, 2010. 120(12):

596 p. $4410-4$. 
597 9. Johnston, J.M., et al., Myeloid Tribbles 1 induces early atherosclerosis via enhanced 598 foam cell expansion. Sci Adv, 2019. 5(10): p. eaax9183.

599 10. Chambers, J.C., et al., Genome-wide association study identifies loci influencing $600 \quad$ concentrations of liver enzymes in plasma. Nat Genet, 2011. 43(11): p. 1131-8.

601 11. Dastani, Z., et al., Novel loci for adiponectin levels and their influence on type 2 diabetes 602 and metabolic traits: a multi-ethnic meta-analysis of 45,891 individuals. PLoS Genet, 603 2012. 8(3): p. e1002607.

604 12. Lihn, A.S., S.B. Pedersen, and B. Richelsen, Adiponectin: action, regulation and 605 association to insulin sensitivity. Obes Rev, 2005. 6(1): p. 13-21.

606 13. Izadi, V., E. Farabad, and L. Azadbakht, Epidemiologic evidence on serum adiponectin level and lipid profile. Int J Prev Med, 2013. 4(2): p. 133-40.

608 14. Xu, A., et al., The fat-derived hormone adiponectin alleviates alcoholic and nonalcoholic fatty liver diseases in mice. J Clin Invest, 2003. 112(1): p. 91-100.

610 15. Pischon, T., et al., Plasma adiponectin levels and risk of myocardial infarction in men. JAMA, 2004. 291(14): p. 1730-7.

612 16. Ostertag, A., et al., Control of adipose tissue inflammation through TRB1. Diabetes, 613 2010. 59(8): p. 1991-2000.

614 17. Eyers, P.A., K. Keeshan, and N. Kannan, Tribbles in the 21st Century: The Evolving Roles of Tribbles Pseudokinases in Biology and Disease. Trends Cell Biol, 2017. 27(4):

617 18. Dedhia, P.H., et al., Differential ability of Tribbles family members to promote degradation of C/EBPalpha and induce acute myelogenous leukemia. Blood, 2010.

620 19. Christy, R.J., et al., Differentiation-induced gene expression in 3T3-L1 preadipocytes: 621 CCAAT/enhancer binding protein interacts with and activates the promoters of two 622 adipocyte-specific genes. Genes Dev, 1989. 3(9): p. 1323-35. 
623 20. Duncan, R.E., et al., Regulation of lipolysis in adipocytes. Annu Rev Nutr, 2007. 27: p.

$624 \quad 79-101$

625 21. Dijk, W. and S. Kersten, Regulation of lipoprotein lipase by Angpt/4. Trends Endocrinol $626 \quad$ Metab, 2014. 25(3): p. 146-55.

627 22. Goldberg, I.J. and M. Merkel, Lipoprotein lipase: physiology, biochemistry, and 628 molecular biology. Front Biosci, 2001. 6: p. D388-405.

629 23. Doolittle, M.H., N. Ehrhardt, and M. Peterfy, Lipase maturation factor 1: structure and 630 role in lipase folding and assembly. Curr Opin Lipidol, 2010. 21(3): p. 198-203.

631 24. Jadhav, K.S. and R.C. Bauer, Trouble With Tribbles-1. Arterioscler Thromb Vasc Biol, 632 2019. 39(6): p. 998-1005.

633 25. Rorth, P., K. Szabo, and G. Texido, The level of C/EBP protein is critical for cell 634 migration during Drosophila oogenesis and is tightly controlled by regulated degradation. 635 Mol Cell, 2000. 6(1): p. 23-30.

636 26. Farmer, S.R., Transcriptional control of adipocyte formation. Cell Metab, 2006. 4(4): p. $637 \quad 263-73$.

638 27. Naiki, T., et al., TRB2, a mouse Tribbles ortholog, suppresses adipocyte differentiation by inhibiting AKT and C/EBPbeta. J Biol Chem, 2007. 282(33): p. 24075-82.

640 28. Madsen, M.S., et al., Peroxisome proliferator-activated receptor gamma and 641 C/EBPalpha synergistically activate key metabolic adipocyte genes by assisted loading. $642 \quad$ Mol Cell Biol, 2014. 34(6): p. 939-54.

643 29. Olofsson, L.E., et al., CCAAT/enhancer binding protein alpha (C/EBPalpha) in adipose 644 tissue regulates genes in lipid and glucose metabolism and a genetic variation in 645 C/EBPalpha is associated with serum levels of triglycerides. J Clin Endocrinol Metab, 646 2008. 93(12): p. 4880-6. 
647 30. Shimada, M., et al., Suppression of diet-induced atherosclerosis in low density lipoprotein receptor knockout mice overexpressing lipoprotein lipase. Proc Natl Acad Sci U S A, 1996. 93(14): p. 7242-6.

650 31. Fan, J., et al., Overexpression of lipoprotein lipase in transgenic rabbits inhibits dietinduced hypercholesterolemia and atherosclerosis. J Biol Chem, 2001. 276(43): p.

653 32. Walton, R.G., et al., Increasing adipocyte lipoprotein lipase improves glucose metabolism in high fat diet-induced obesity. J Biol Chem, 2015. 290(18): p. 11547-56.

655 33. Koster, A., et al., Transgenic angiopoietin-like (angptl)4 overexpression and targeted 656 disruption of angpt/4 and angpt/3: regulation of triglyceride metabolism. Endocrinology, 2005. 146(11): p. 4943-50.

658 34. Aryal, B., et al., Absence of ANGPTL4 in adipose tissue improves glucose tolerance and attenuates atherogenesis. JCI Insight, 2018. 3(6).

660 35. Aviram, M., E.L. Bierman, and A. Chait, Modification of low density lipoprotein by lipoprotein lipase or hepatic lipase induces enhanced uptake and cholesterol

663 36. Sehayek, E., U. Lewin-Velvert, T. Chajek-Shaul, and S. Eisenberg, Lipolysis exposes 664 unreactive endogenous apolipoprotein E-3 in human and rat plasma very low density 665 lipoprotein. J Clin Invest, 1991. 88(2): p. 553-60.

666 37. Tilg, H. and A.R. Moschen, Adipocytokines: mediators linking adipose tissue, 667 inflammation and immunity. Nat Rev Immunol, 2006. 6(10): p. 772-83.

668 38. Yanai, H. and H. Yoshida, Beneficial Effects of Adiponectin on Glucose and Lipid 669 Metabolism and Atherosclerotic Progression: Mechanisms and Perspectives. Int J Mol 670 Sci, 2019. 20(5). 
671 39. Bauche, I.B., et al., Overexpression of adiponectin targeted to adipose tissue in

672 transgenic mice: impaired adipocyte differentiation. Endocrinology, 2007. 148(4): p.

$673 \quad 1539-49$.

674 40. Christou, G.A. and D.N. Kiortsis, Adiponectin and lipoprotein metabolism. Obes Rev, 675 2013. 14(12): p. 939-49.

676 41. Combs, T.P., et al., A transgenic mouse with a deletion in the collagenous domain of 677 adiponectin displays elevated circulating adiponectin and improved insulin sensitivity.

$678 \quad$ Endocrinology, 2004. 145(1): p. 367-83.

679 42. Lagor, W.R., et al., Deletion of murine Arv1 results in a lean phenotype with increased 680 energy expenditure. Nutr Diabetes, 2015. 5: p. e181.

681 43. Reimand, J., et al., Pathway enrichment analysis and visualization of omics data using 682 g:Profiler, GSEA, Cytoscape and EnrichmentMap. Nat Protoc, 2019. 14(2): p. 482-517.

683 44. Kulak, N.A., et al., Minimal, encapsulated proteomic-sample processing applied to copy684 number estimation in eukaryotic cells. Nat Methods, 2014. 11(3): p. 319-24.

685 45. Bruderer, R., et al., Extending the limits of quantitative proteome profiling with data686 independent acquisition and application to acetaminophen-treated three-dimensional 687 liver microtissues. Mol Cell Proteomics, 2015. 14(5): p. 1400-10.

688 46. Basu, D., J. Manjur, and W. Jin, Determination of lipoprotein lipase activity using a novel 689 fluorescent lipase assay. J Lipid Res, 2011. 52(4): p. 826-32. 
Figures:
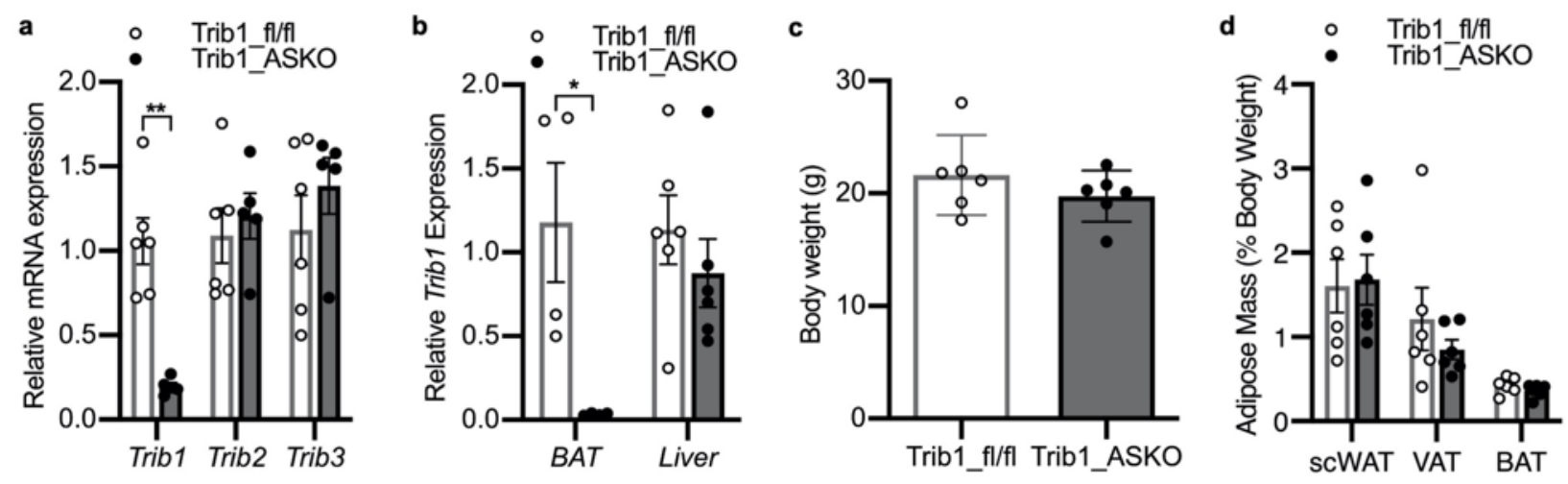

e
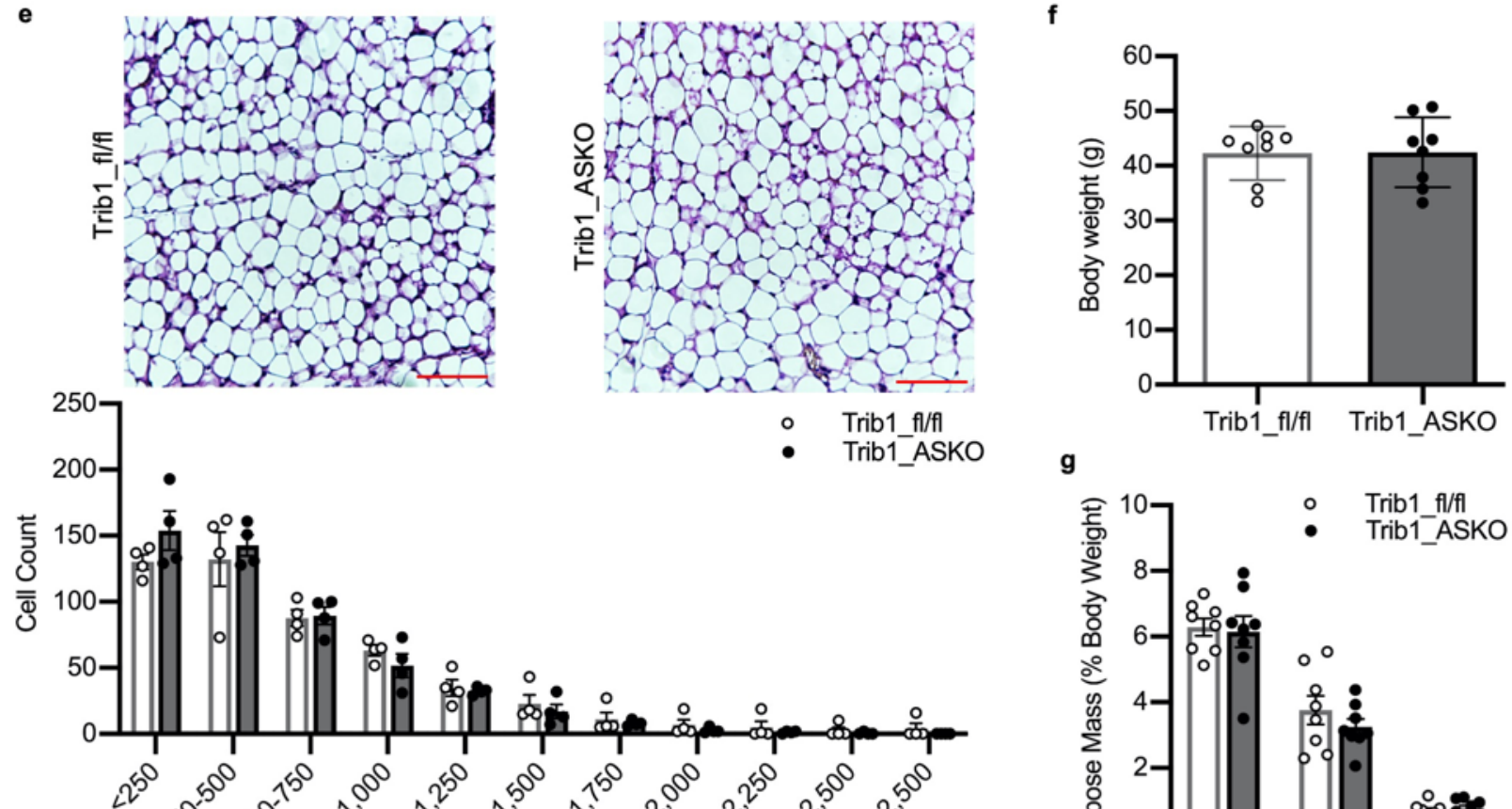

- Trib1_ASKO

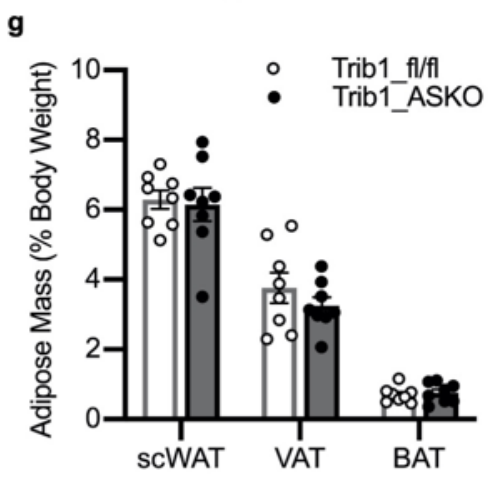

3 Figure 1: Adipocyte-specific knockout of Tribbles1 does not result in defects in adiposity. a, Taqman qPCR for Trib1, Trib2, and Trib3 in scWAT from 8-10-week-old Trib1_fl/fl and ASKO mice $(n=5)$. b, Taqman qPCR for Trib1 from BAT $(n=4)$ and livers $(n=6)$ of Trib1_fl/fl and Trib1_ASKO mice. c,d, Body weight (c) and adipose depot masses (d) in chowfed Trib1_fl/fl and ASKO mice $(n=6)$. e, Representative H\&E stain of scWAT from Trib1_fl/fl and ASKO mice and quantitation of cell size by Adiposoft ( $n=4$ mice). Bar $=100 \mu \mathrm{m} . \mathbf{f}, \mathbf{g}$, Body weight (f) and adipose depot masses $(\mathbf{g})$ in 12 week HFD-fed Trib1_fl/fl and ASKO mice $(n=8)$. 
10 All gene expression data is depicted as mean \pm s.e.m. All other data is depicted as mean \pm s.d.

11 Significance in all panels determined by Student's $t$ test $\left({ }^{*} p<0.05,{ }^{* *} p<0.01\right)$.

12

13

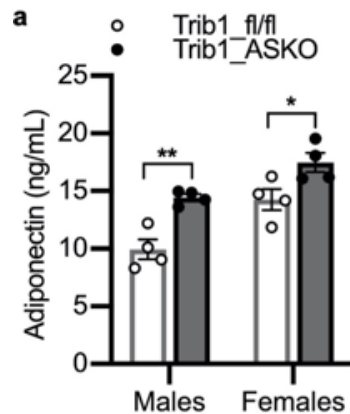

e

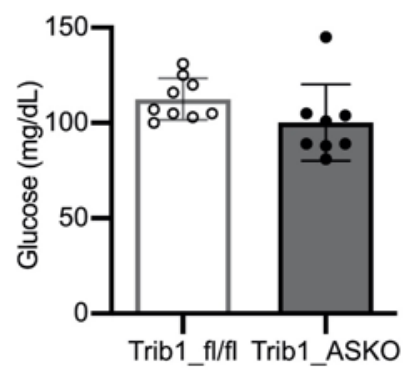

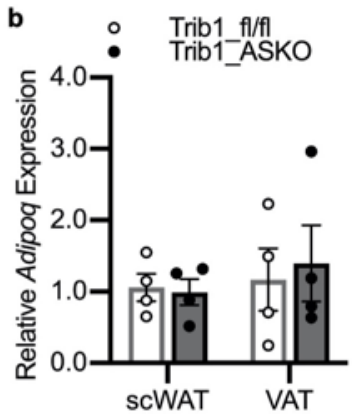

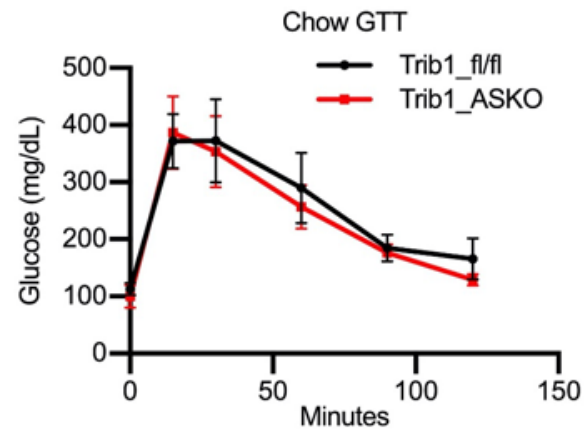

d

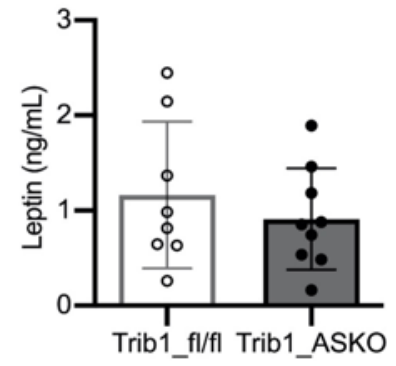

Chow GTT

f $\quad: \quad$ Trib1_fl/fl ${ }_{\text {Trib1_ASKO }} \mathbf{g}$
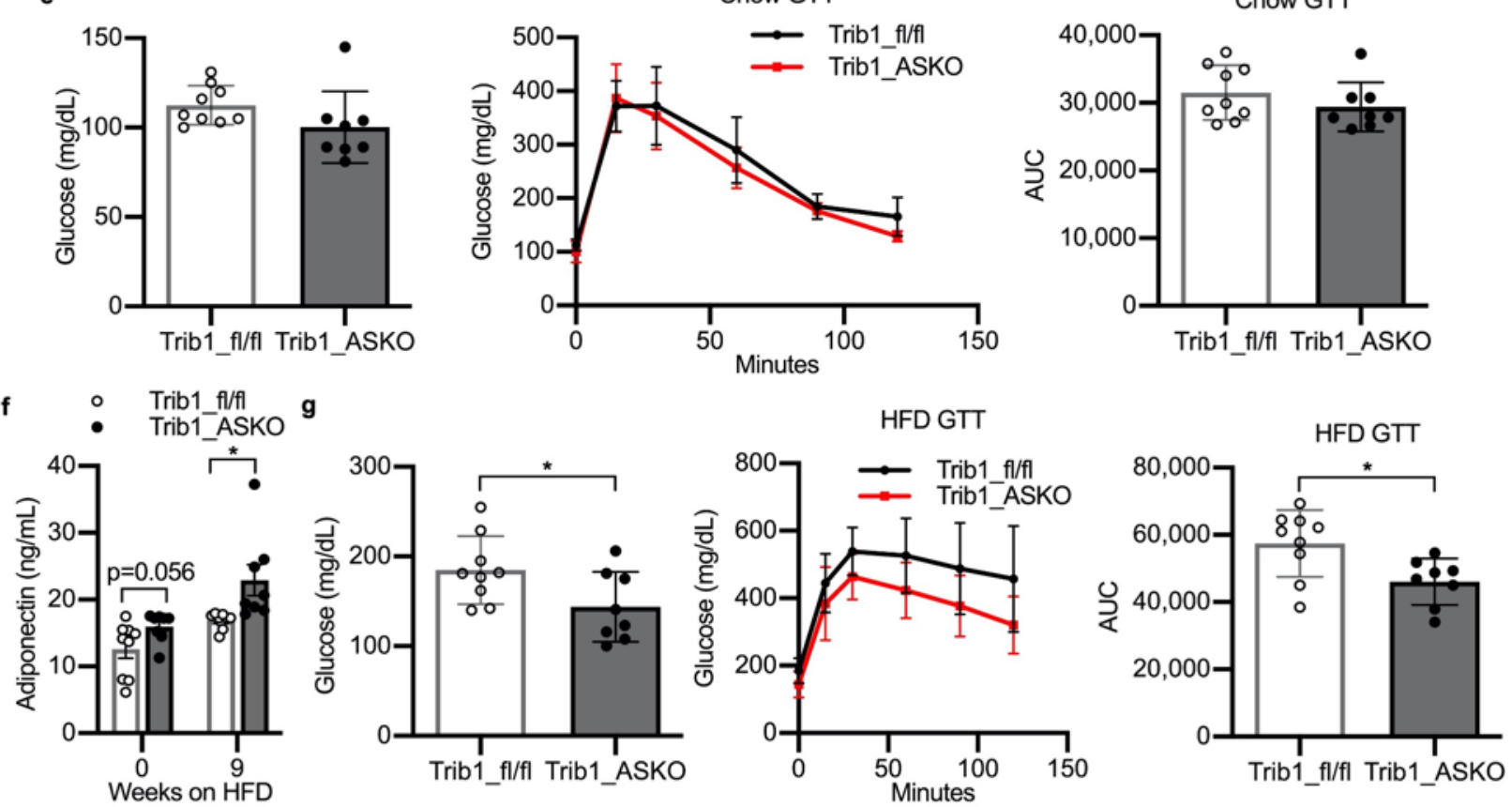

15 Figure 2: Trib1_ASKO mice have increased plasma adiponectin. a, Plasma adiponectin in

16 4hr-fasted 8-week-old chow-fed Trib1_fl/fl and Trib1_ASKO mice $(n=4)$. b, Taqman qPCR for

17 Adipog in scWAT and VAT from Trib1_fl/fl and Trib1_ASKO mice $(n=4)$. c,d, Plasma resistin

18 (c) and plasma leptin (d) in $4 \mathrm{hr}$-fasted chow-fed Trib1_fl/fl and Trib1_ASKO mice $(n=8)$. e,

19 Glycemic traits after $16 \mathrm{hr}$ overnight fast in chow-fed 8-10-week-old male Trib1_fl/fl and

20 Trib1_ASKO mice $(n=7)$. f, Plasma adiponectin in $4 \mathrm{hr}$-fasted 9 week HFD-fed Trib1_fl/fl and 
21 Trib1_ASKO mice $(n=8) . \mathbf{g}$, Glycemic traits after $16 \mathrm{hr}$ overnight fast in 12 week HFD-fed male

22 Trib1_fl/fl and Trib1_ASKO mice $(n=8)$. Gene expression is depicted as mean \pm s.e.m. All

23 other data is depicted as mean \pm s.d. Significance in all panels determined by Student's $t$ test

$24 \quad\left({ }^{*} p<0.05,{ }^{* *} p<0.01,{ }^{* * *} p<0.0001\right)$.

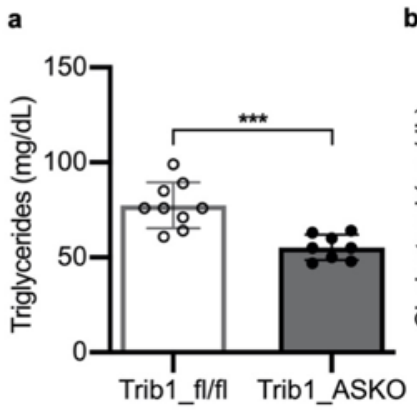

c
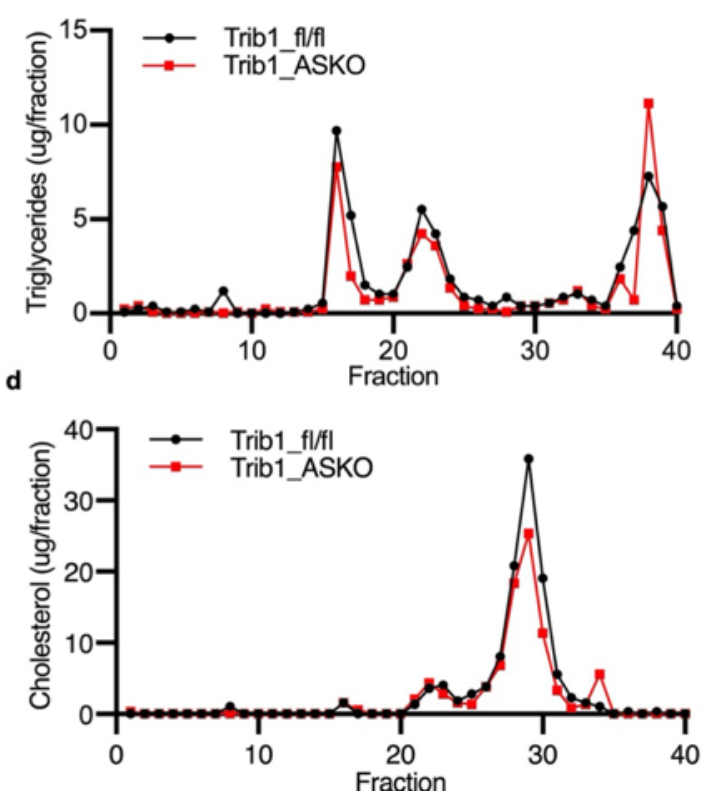

b

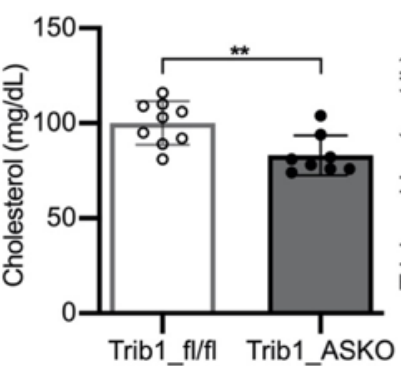

$$
\text { g }
$$

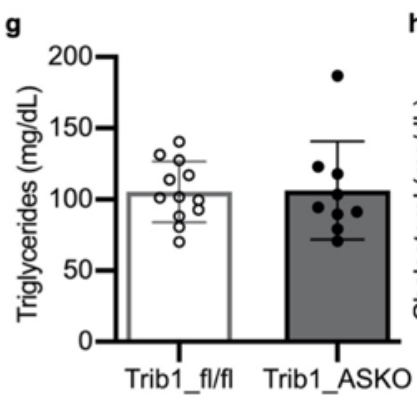

$\mathbf{i}$

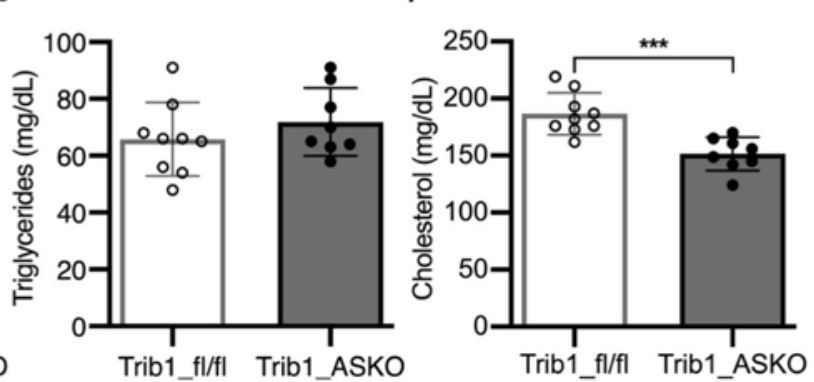

h
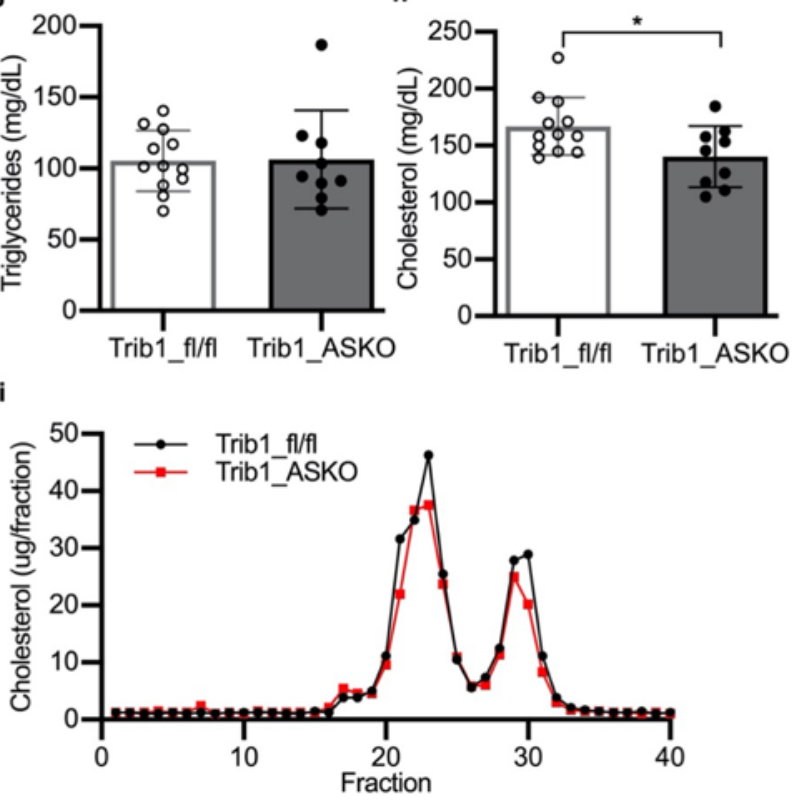

Figure 3: Trib1_ASKO mice have decreased plasma cholesterol and triglycerides. a,b,

28 Plasma triglyceride (a) and total cholesterol (b) levels in 8-10-week-old, $4 \mathrm{hr}$-fasted chow-fed

29 male Trib1_fl/fl and Trib1_ASKO mice $(n=8) . \mathbf{c}, \mathbf{d}$, Plasma triglyceride $(\mathbf{c})$ and cholesterol $(\mathbf{d})$

30 FPLC profiles of pooled plasma $(n=4)$ from 4 hr-fasted chow-fed male Trib1_fl/fl and

31 Trib1_ASKO mice. e,f, Plasma triglyceride (e) and total cholesterol (f) levels in 4 hr-fasted 12

32 week HFD-fed Trib1_fl/fl and Trib1_ASKO mice $(n=8)$. g,h, Plasma triglyceride(g) and total 
33 cholesterol (h) levels in 8-week-old, 4 hr-fasted chow-fed male Trib1_fl/fl ; Ldlr KO and

34 Trib1_ASKO ; Ldlr KO mice $(n=9)$. i, Cholesterol FPLC profile of pooled plasma $(n=4)$ from 4

35 hr-fasted chow-fed female Trib1_fl/fl ; Ldlr KO and Trib1_ASKO ; Ldlr KO mice. Data is depicted

36 as mean \pm s.d. Significance in all panels determined by Student's $t$ test $\left({ }^{*} p<0.05,{ }^{* *} p<0.01\right.$

$\left.37^{* * *} p<0.001\right)$.

38

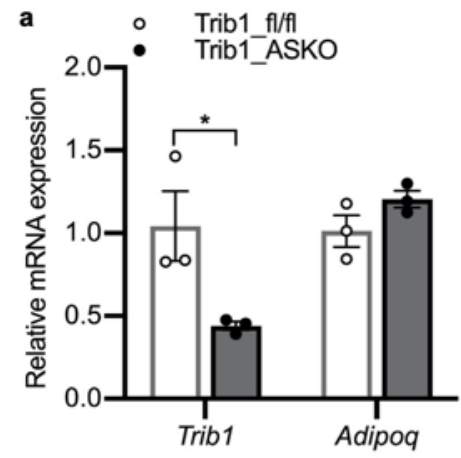

d

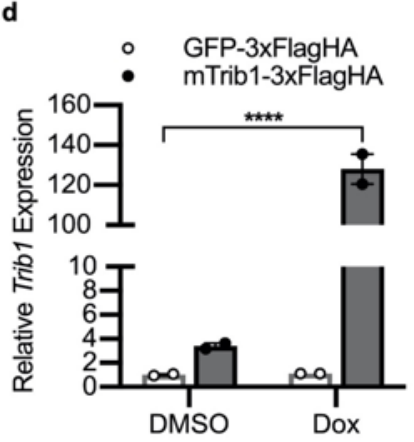

b

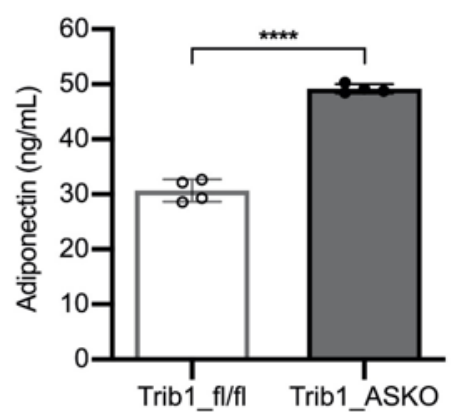

e

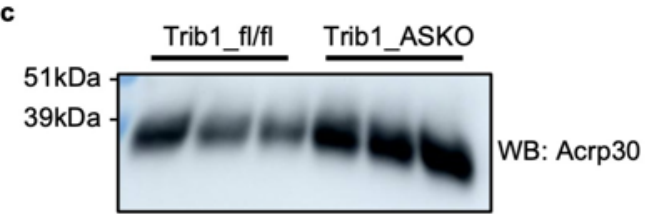

$51 \mathrm{kDa}$

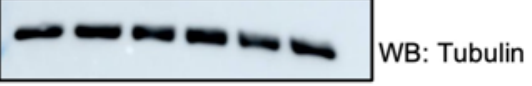

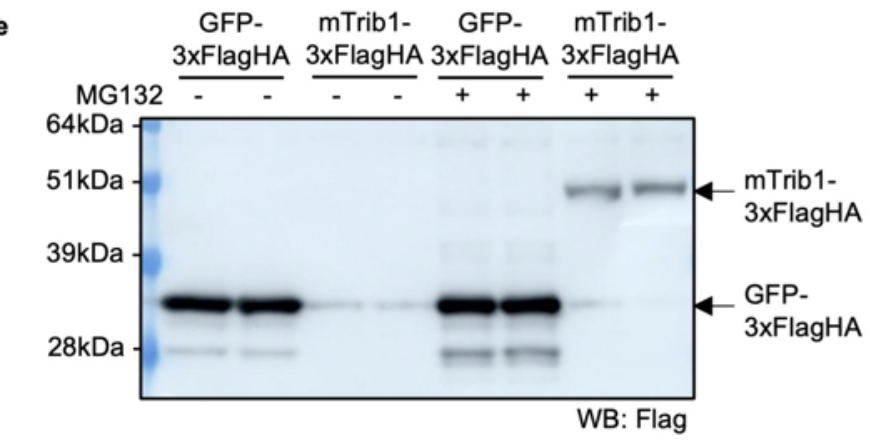

f

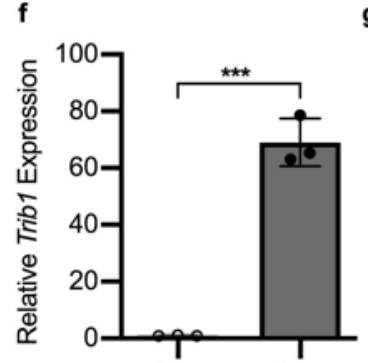

g
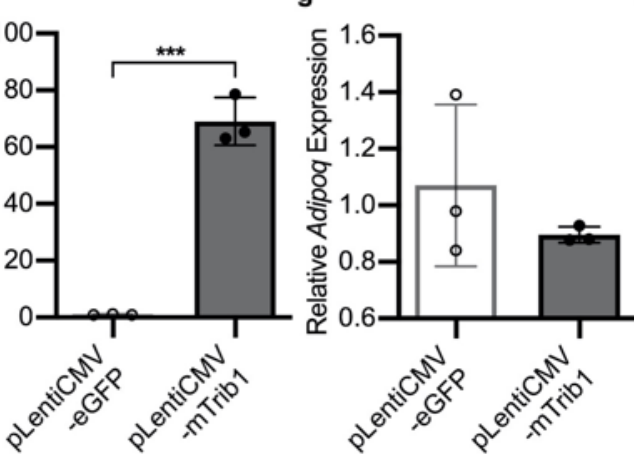

h

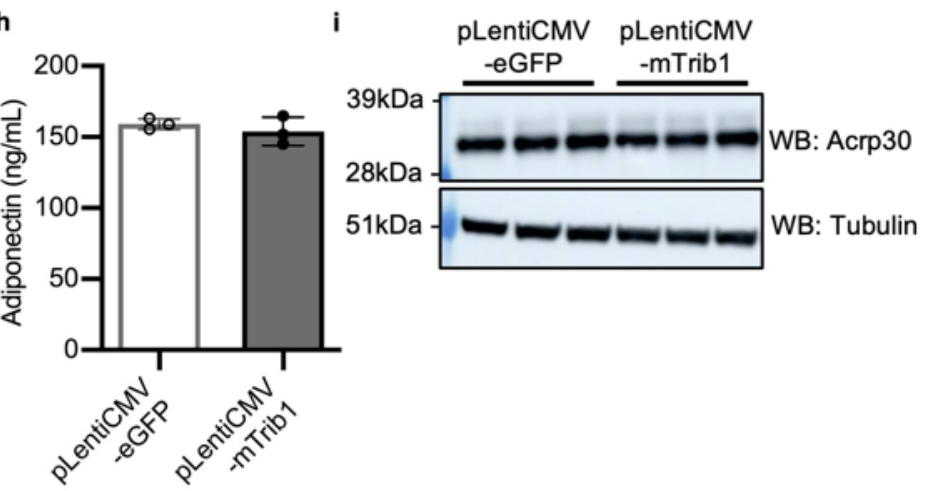

41 secretion. a, Taqman qPCR for Trib1 and Adipoq in SVF-derived adipocytes $(n=3)$. b,

42 Adiponectin concentration in conditioned media from SVF-derived adipocytes $(n=4)$. 
43 Conditioned media was generated by culturing SVF-derived adipocytes in OptiMEM reduced-

44 serum media for $4 \mathrm{hr}$. c, Western blot analysis of adiponectin (Acrp30) and tubulin levels in

45 SVF-derived adipocytes. d, Taqman qPCR for Trib1 in pSlik-neo-TTMCS_eGFP-3xFlagHA and

46 pSlik-neo-TTMCS_mTrib1-3xFlagHA stable 3T3-L1 cells treated with either DMSO or

47 doxycycline $(1 \mu \mathrm{g} / \mathrm{mL})(n=2)$. Gene expression is expressed relative to the DMSO-treated GFP

48 stable cells. Significance relative to DMSO treated GFP stable cells was determined by 1-way

49 ANOVA (Dunnett's multiple comparison test) e, Western blot for Flag-tagged protein

50 overexpression in pSlik-neo-TTMCS_eGFP-3xFlagHA and pSlik-neo-TTMCS_mTrib1-3xFlagHA

51 stable 3T3-L1 preadipocytes induced with $1 \mu \mathrm{g} / \mathrm{mL}$ Dox for $48 \mathrm{hr}$ and treated with or without 20

$52 \mu \mathrm{M}$ MG132 for $5 \mathrm{hr}$. f-i, Mature 3T3-L1 adipocytes were transduced with lentivirus to

53 overexpress eGFP (pLentiCMV-eGFP) or mTrib1 (pLentiCMV-mTrib1) under the CMV

54 promoter. Taqman qPCR for Trib1 (f) and Adipoq $(\mathbf{g})(n=3)$. Gene expression is expressed

55 relative to the pLentiCMV-eGFP group. $\mathbf{h}$, ELISA for adiponectin in $4 \mathrm{hr}$ conditioned media $(n=$

56 3). i, Western blot for adiponectin protein expression. Gene expression is depicted as mean \pm

57 s.e.m. All other data is depicted as mean \pm s.d. Significance in all panels determined by

58 Student's $t$ test except where indicated $\left({ }^{*} p<0.05,{ }^{* * *} p<0.001,{ }^{* * *} p<0.0001\right)$. 
a

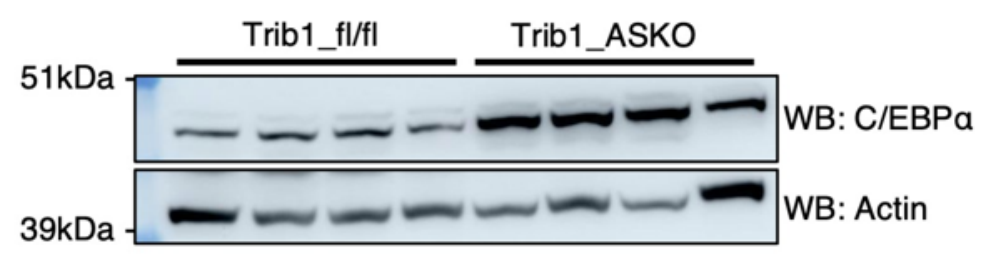

b

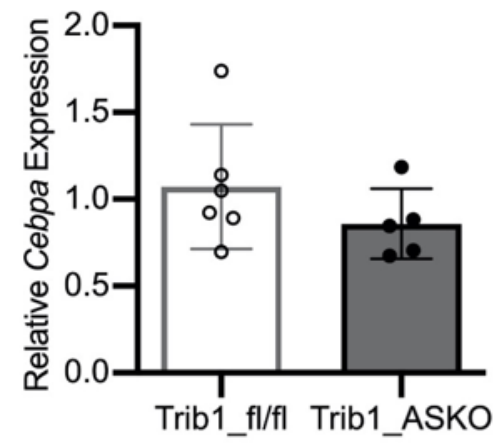

d

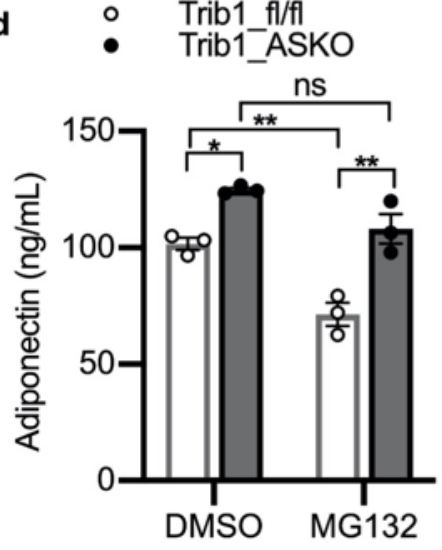

c

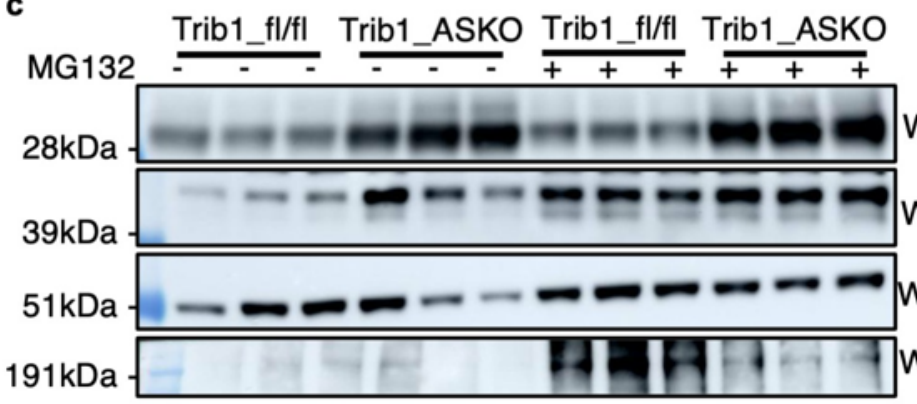

WB: Acrp30

WB: C/EBPa

WB: Tubulin

WB: Ubiquitin

71 Figure 5: Trib1 does not regulate adiponectin through the proteasome in SVF-derived

72 adipocytes. a, Western blot of C/EBPa in scWAT of Trib1_fl/fl and Trib1_ASKO mice. b, qPCR

73 for Cebpa gene expression in scWAT of Trib1_fl/fl and Trib1_ASKO mice $(n=5)$. c,d, SVF-

74 derived adipocytes from Trib1_fl/fl and Trib1_ASKO scWAT were differentiated, pretreated with

$7530 \mu \mathrm{M}$ MG132 for $1 \mathrm{hr}$, and then treated with $30 \mu \mathrm{M}$ MG132 for an additional $4 \mathrm{hr}$ before

76 measuring protein expression and adiponectin secretion. c, Western blot for adiponectin

77 (Acrp30) and C/EBPa protein in $5 \mathrm{hr}$ MG132 treated Trib1_fl/fl and Trib1_ASKO adipocytes. d,

78 ELISA for adiponectin in $4 \mathrm{hr}$ conditioned media from $30 \mu \mathrm{M}$ MG132 treated Trib1_fl/fl and

79 Trib1_ASKO adipocytes $(n=3)$. Gene expression is depicted as mean \pm s.e.m. All other data is

80 depicted as mean \pm s.d. Significance in (b) determined by Student's $t$ test, and significance in

81 (d) by 2-way ANOVA (Tukey's multiple correction) ( $\mathrm{ns}=$ not significant, ${ }^{*} \mathrm{p}<0.05,{ }^{* *} \mathrm{p}<0.01$ ). 
a

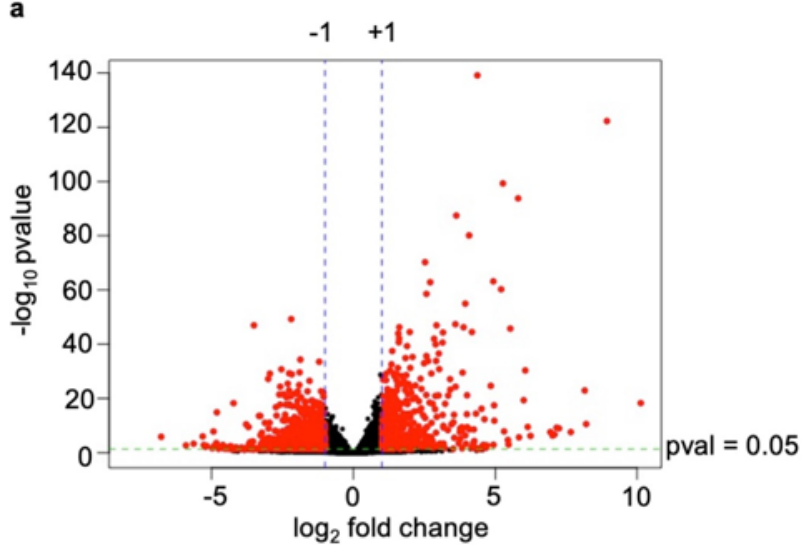

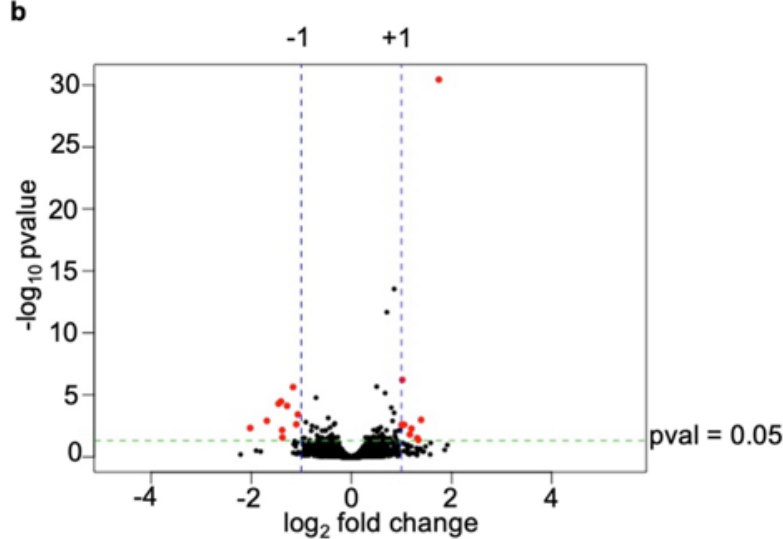

c

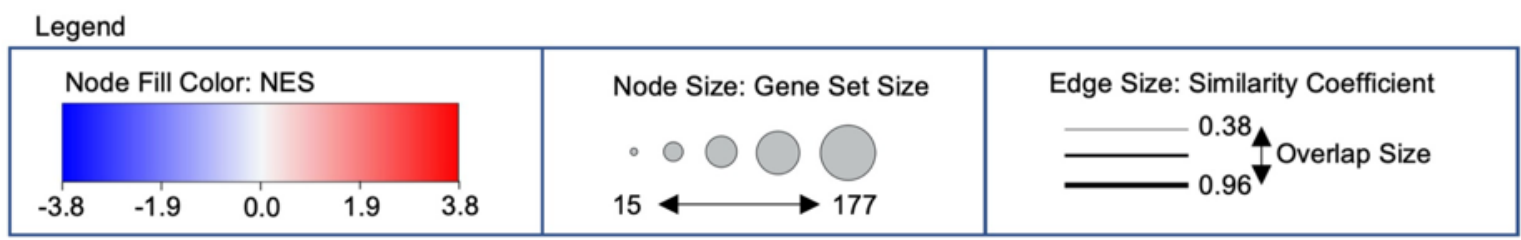

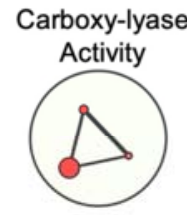

Transition lon Transport

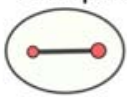

$\mathrm{CH}-\mathrm{OH}$ Reduction

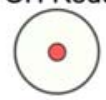

$\mathrm{CH}-\mathrm{CH}$ Reduction

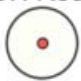

Monosaccharide

Biosynthetic Process
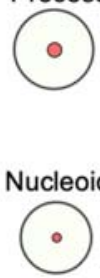

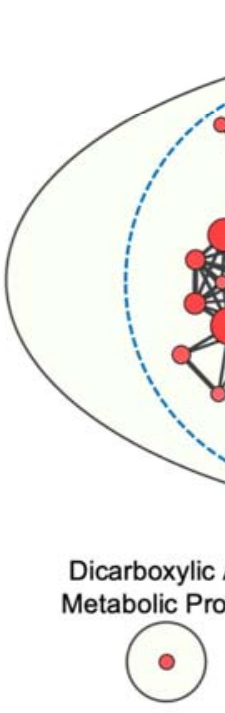

Carboxylic Ester Hydrolase Activity

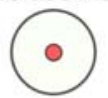

Endoplasmic Reticulum Membrane

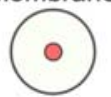

Thiolester Hydrolase Activity

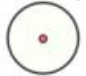

Ribosome Biogenesis

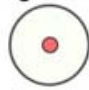

Figure

6: Trib1_ASKO adipocytes have widespread transcriptiona changes in
Extracellular Matrix

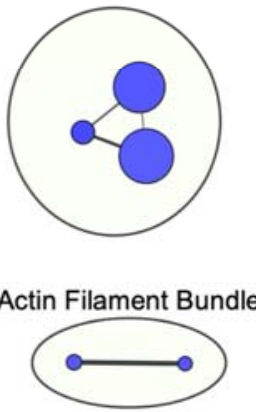

Embryonic Organ Development

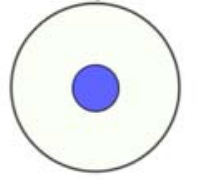

Endothelial Cell Proliferation
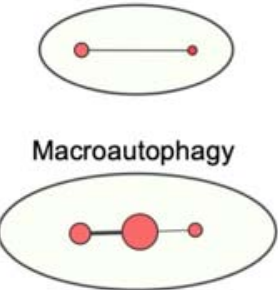

Positive Regulation of Leukocyte Production

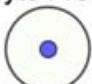

84 mitochondrial and lipid metabolism pathways. a, Volcano plot of DESeq2 analysis of RNA- 
85 seq data from adipocytes isolated from scWAT from Trib1_fl/fl and Trib1_ASKO mice $(n=4)$. b,

86 Volcano plot of DESeq2 analysis of RNA-seq data from hepatocytes from Trib1_fl/fl and

87 Trib1_ASKO mice $(n=4)$. c, Cytoscape enrichment plot of Gene Set Enrichment Analysis

88 (GSEA) of differentially expressed adipocyte genes (padj<0.05). Enrichment analysis and

89 clustering were performed as described in the Methods section. Clusters upregulated in

90 Trib1_ASKO samples are shown in red, and clusters upregulated in Trib1_fl/fl samples are

91 shown in blue. Dashed blue outlines indicate larger clusters that were further subclustered

92 manually to facilitate interpretation. NES = normalized enrichment score.

93

94

a

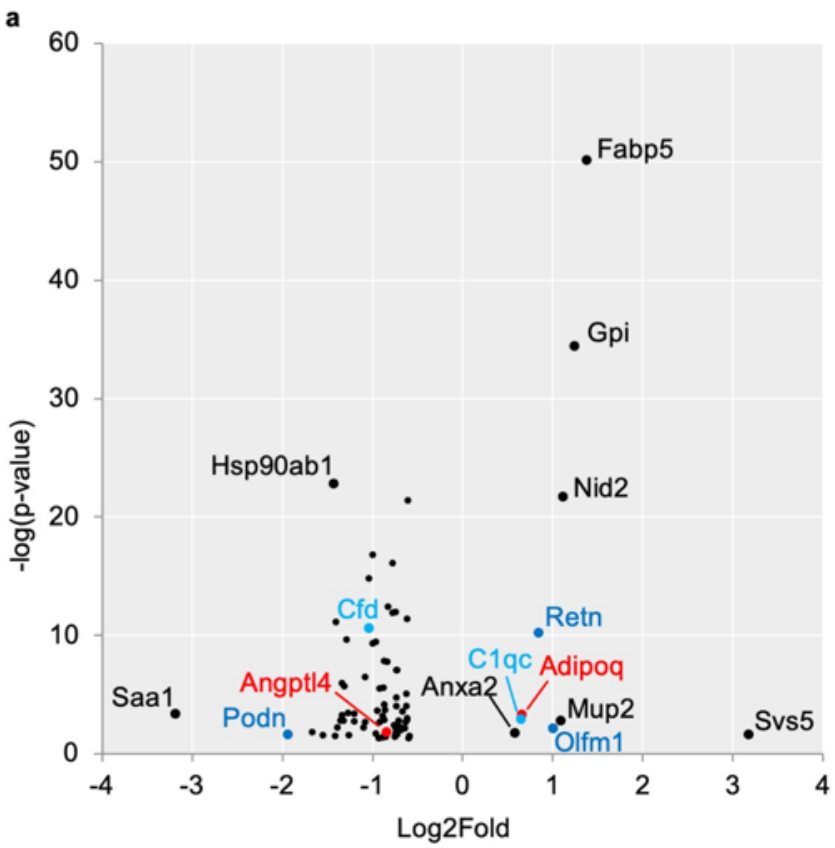

b

\begin{tabular}{ccc} 
Gene & $\begin{array}{c}\text { Log2Fold } \\
\text { Change }\end{array}$ & FDR \\
\hline Lpl & 0.855 & $1.11 \mathrm{E}-07$ \\
\hline Angpt/4 & -0.591 & 0.004 \\
Lmf1 & 0.657 & $3.11 \mathrm{E}-06$
\end{tabular}

c

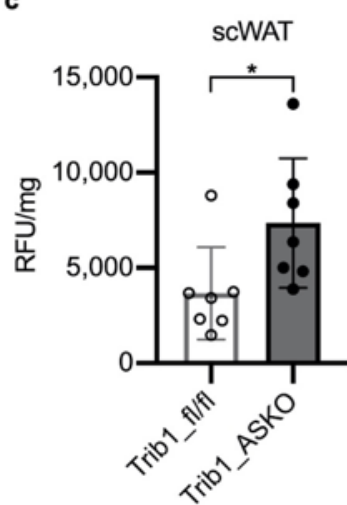

d

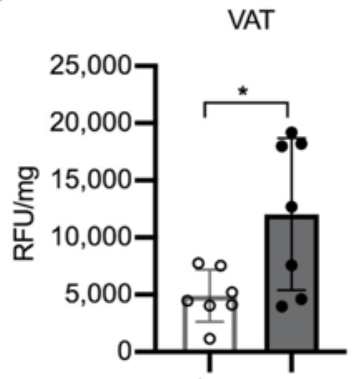

96 Figure 7: Trib1_ASKO mice have increased adipose tissue lipoprotein lipase activity. a,

97 DIA proteomics data of conditioned media from Trib1_ASKO vs. Trib1_fl/fl scWAT explants $(n=$

98 3). Differential secretion was determined by Spectronaut analysis and results were filtered for

99 secreted proteins (Uniprot keywords). Size and color of datapoints are for facilitating

100 visualization. b, DESeq2 results for $L p l$, Angpt/4, and $L m f 1$ from RNA-seq of Trib1_fl/fl and 

available under aCC-BY-NC-ND 4.0 International license.

101 Trib1_ASKO adipocytes. c,d, Lpl activity in scWAT (c) and VAT (d) extracts $(n=5)$. Data

102 depicted as mean \pm s.d. Significance in $\mathbf{c}, \mathbf{d}$ determined by Student's $t$ test $\left({ }^{*} \mathrm{p}<0.05\right)$. 